\title{
DISCOVERY OF A WIDE PLANETARY-MASS COMPANION TO THE YOUNG M3 STAR GU PSC
}

\author{
Marie-Eve Naud ${ }^{1}$, Étienne Artigau $^{1}$, Lison Malo $^{1}$, LoḮ Albert $^{1}$, René Doyon $^{1}$, David Lafrenière ${ }^{1}$, \\ Jonathan Gagné ${ }^{1}$, Didier Saumon ${ }^{2}$, Caroline V. Morley ${ }^{3}$, France Allard ${ }^{4}$, Derek Homeier ${ }^{4}$, \\ Charles A. Beichman ${ }^{5}$, Christopher R. Gelino ${ }^{5,6}$, and Anne Boucher ${ }^{1}$ \\ ${ }^{1}$ Département de physique and Observatoire du Mont-Mégantic, Université de Montréal, Montréal H3C 3J7, Canada; \\ naud@astro.umontreal.ca \\ 2 Los Alamos National Laboratory, Los Alamos, NM 87545, USA \\ ${ }^{3}$ Department of Astronomy and Astrophysics, University of California, Santa Cruz, CA 95064, USA \\ ${ }^{4}$ Centre de Recherche Astrophysique de Lyon, UMR 5574 CNRS, Université de Lyon, École Normale Supérieure \\ de Lyon, 46 Allée d'Italie, F-69364 Lyon Cedex 07, France \\ ${ }^{5}$ Infrared Processing and Analysis Center, MS 100-22, California Institute of Technology, Pasadena, CA 91125, USA \\ ${ }^{6}$ NASA Exoplanet Science Institute, California Institute of Technology, 770 S. Wilson Ave., Pasadena, CA 91125, USA \\ Received 2013 October 31; accepted 2014 March 26; published 2014 April 28
}

\begin{abstract}
We present the discovery of a comoving planetary-mass companion $\sim 42^{\prime \prime}(\sim 2000 \mathrm{AU})$ from a young M3 star, GU Psc, a likely member of the young AB Doradus Moving Group (ABDMG). The companion was first identified via its distinctively red $i-z$ color $(>3.5)$ through a survey made with Gemini-S/GMOS. Follow-up Canada-France-Hawaii Telescope/WIRCam near-infrared (NIR) imaging, Gemini-N/GNIRS NIR spectroscopy and Wide-field Infrared Survey Explorer photometry indicate a spectral type of T3.5 \pm 1 and reveal signs of low gravity which we attribute to youth. Keck/Adaptive Optics NIR observations did not resolve the companion as a binary. A comparison with atmosphere models indicates $T_{\text {eff }}=1000-1100 \mathrm{~K}$ and $\log g=4.5-5.0$. Based on evolution models, this temperature corresponds to a mass of 9-13 $M_{\text {Jup }}$ for the age of ABDMG (70-130 Myr). The relatively well-constrained age of this companion and its very large angular separation to its host star will allow its thorough characterization and will make it a valuable comparison for planetary-mass companions that will be uncovered by forthcoming planet-finder instruments such as Gemini Planet Imager and SPHERE.
\end{abstract}

Key words: infrared: planetary systems - planetary systems - planets and satellites: detection - stars: imaging stars: individual (GU Psc) - stars: low-mass

Online-only material: color figures

\section{INTRODUCTION}

Of the nearly thousand of exoplanets known so far, the majority (>90\%) were detected through the radial velocity and the transit methods. ${ }^{7}$ The sample is thus biased toward planets at relatively small orbital separations of a few astronomical units or less. Direct imaging complements these methods by finding the most massive planets at large orbital separations. Most of the directly imaged planets known today (e.g., HR 8799bcde, Marois et al. 2008, 2010; $\beta$ Pictoris b, Lagrange et al. 2009; GJ 504 b, Kuzuhara et al. 2013; HD 95086 b, Rameau et al. 2013) were found using high-contrast imaging techniques and Adaptive Optics (AO).

The recent discoveries of low-mass companions at very large orbital separations through seeing-limited imaging came somewhat as a surprise and provided new constraints to formation models. For example, Ross 458(AB) c is a late T dwarf located $1100 \mathrm{AU}\left(102^{\prime \prime}\right)$ from its parent pair of M dwarfs (Goldman et al. 2010). Its estimated mass is below the deuterium-burning limit ( $\sim 13 M_{\text {Jup }}$; Spiegel et al. 2011), a criteria commonly used as the delineation between planets and brown dwarfs. HN Peg b is a more massive (22 $\left.\pm 9 M_{\text {Jup }}\right)$ T2 companion located $795 \mathrm{AU}$ (43"'2) from a G dwarf of $0.3 \pm 0.2$ Gyr (Luhman et al. 2007). The planetary-mass companion to the young (8-20 Myr) brown dwarf 2MASS J12073346-3932539 (2M1207 hereafter; Gizis 2002; Chauvin et al. 2004; Ducourant et al. 2008) could also be

\footnotetext{
7 The Extrasolar Planets Encyclopaedia (Schneider et al. 2011),
} http://exoplanet.eu/. added as an example. For a more complete list, see Neuhäuser \& Schmidt (2012).

The wide orbital distances of these companions preclude in situ formation in a protoplanetary disk, which is normally expected for planets. They could have been ejected at this distance through dynamical interactions, or formed like brown dwarfs and stars through collapse and fragmentation of a molecular cloud core. These distant objects are not only easier to detect but also easier to study spectroscopically. They thus constitute excellent proxies to improve atmospheric models and better understand closer-in companions found with AO. For example, the study of Ross 458 (AB) c (Burningham et al. 2011; Burgasser et al. 2010b) suggested that condensate opacity plays a role in the spectra of late $\mathrm{T}$ dwarfs and showed that including them allows a better determination of the physical parameters of these objects. Such a detailed spectroscopic characterization is very challenging for planetary-mass companion found very close to their parent star (e.g., HR 8799b and c; Barman et al. 2011; Konopacky et al. 2013).

Young stars are really interesting for direct imaging because their planets, still contracting, are hotter and more luminous than their older counterpart and thus are easier to detect. In the solar neighborhood, young stars are often found in young moving groups and associations (Zuckerman \& Song 2004). These groups of stars share a common origin and thus have similar positions and space motions in the Galaxy. Since the determination of the mass of directly imaged companions relies on the use of evolutionary models, these young association members, with their constrained age, are prime targets for direct 
imaging investigation. The lower-mass members of those groups are great targets for imaging, their faint luminosity results in a higher contrast for a planet of a given mass.

In 2011, we initiated a survey with Gemini-South/GMOS (Hook et al. 2004) to search for low-mass companions via their distinctively red $i-z$ colors around candidate members of nearby young ( $<150 \mathrm{Myr}$ ) associations (É. Artigau et al., in preparation). The 91 targets of this survey are low-mass stars (K5-M5) that were recently identified as likely members of nearby young moving groups by Malo et al. (2013) through a novel Bayesian analysis. This survey allowed to search for companion $\gtrsim 8 \quad M_{\text {Jup }}$ at separations ranging from $\sim 300$ to $5000 \mathrm{AU}$. One single candidate companion was identified, around GU Pisces (hereafter GU Psc), an M3 \pm 0.5 star candidate member of the young AB Doradus Moving Group (ABDMG; Zuckerman et al. 2004, 2011).

In this paper, we present new observations of both the host star, GU Psc, and its newly detected companion, showing that they form a bound system, with an age consistent with that of the ABDMG, which suggests, according to evolutionary models, a companion mass below the deuterium-burning limit. In Section 2, we present various observations that were carried on the host star, and then on the companion, and explain the reduction of the associated data. In Section 3, the physical properties of the host star and of the companion are derived. Finally, in Section 4, the stability of this wide pair and plausible formation scenarios are briefly discussed, and the interest of the companion as a proxy for other, closer-in companions is presented.

\section{OBSERVATION AND DATA REDUCTION}

All astrometric and photometric measurements for both the host star and the companion are reported in Table 1 . We present near-infrared (NIR) photometry in the Mauna Kea Observatory filter set standard (MKO; Simons \& Tokunaga 2002; Tokunaga $\&$ Vacca 2005) unless stated otherwise.

\subsection{The Host Star, GU Psc}

\subsubsection{High-resolution Spectroscopy}

High-resolution optical spectroscopy was obtained with ESPaDOnS (Donati et al. 2006) at the Canada-France-Hawaii Telescope (CFHT). The data were reduced by the CFHT queue service observing team using the pipeline UPENA 1.0, that uses the Libre-ESpRIT software (Donati et al. 1997). The resulting spectrum goes from $0.37 \mu \mathrm{m}$ to $1.05 \mu \mathrm{m}$ (40 grating orders) with $R \sim 68,000$.

High-resolution spectroscopy was also acquired for GU Psc with two different instruments in the NIR with the specific goal of measuring its precise radial velocity. With CRIRES on the Very Large Telescope (VLT; Kaeufl et al. 2004), the 0".4-wide slit was used in an order centered on $1.555 \mu \mathrm{m}$ for a resulting $R \sim 50,000$. With PHOENIX on Gemini-South (Hinkle et al. 2003), we used the 0 '.34 slit with the 1.547-1.568 $\mu$ m blocking filter and obtained a resolving power of $R \sim 52,000$. The NIR spectroscopic data were reduced using standard procedures with a custom IDL pipeline.

\subsubsection{Near-infrared Medium-resolution Spectroscopy}

A $0.8-2.4 \mu \mathrm{m}$ NIR spectrum of the primary was also obtained with SpeX, the medium-resolution spectrograph and imager at NASA InfraRed Telescope Facility (Rayner et al. 2003), using
Table 1

Properties of the GU Psc System

\begin{tabular}{lcc}
\hline \hline Property & GU Psc & GU Psc b \\
\hline Spectral type & M3 $\pm 0.5^{\mathrm{a}}$ & T3.5 \pm 1 \\
Age & $100 \pm 30 \mathrm{Myr}^{\mathrm{b}}$ \\
Distance & $48 \pm 5 \mathrm{pc}^{\mathrm{c}}$ \\
Ang. sep. & \multicolumn{2}{c}{$41.97 \pm 00^{\prime} 03^{\mathrm{d}}$} \\
Proj. phys. sep. & \multicolumn{2}{c}{$2000 \pm 200 \mathrm{AU}$} \\
\hline
\end{tabular}

\begin{tabular}{|c|c|c|}
\hline $\begin{array}{l}\text { R.A. (J2000) } \\
\text { (h m s) }\end{array}$ & 011235.04 & $011236.48^{\mathrm{d}}$ \\
\hline $\begin{array}{l}\text { Decl. (J2000) } \\
\text { (d m s) }\end{array}$ & +170355.7 & +170431.8 \\
\hline$\mu_{\alpha} \cos \delta, \mu_{\delta}\left(\operatorname{mas~yr}^{-1}\right)$ & $90 \pm 6,-102 \pm 6^{\mathrm{e}}$ & $98 \pm 15,-92 \pm 15^{\mathrm{f}}$ \\
\hline$B(\mathrm{mag})$ & $15.30^{\mathrm{g}}$ & \\
\hline$V(\mathrm{mag})$ & $13.55^{\mathrm{h}}$ & \\
\hline$R$ (mag) & $12.92^{\mathrm{g}}$ & \\
\hline$u^{\prime}(\mathrm{mag})$ & $17.347 \pm 0.011^{\mathrm{i}}$ & \\
\hline$g^{\prime}(\mathrm{mag})$ & $15.499 \pm 0.005^{\mathrm{i}}$ & \\
\hline$r^{\prime}(\mathrm{mag})$ & $13.650 \pm 0.003^{\mathrm{i}}$ & \\
\hline$i^{\prime}(\mathrm{mag})$ & $12.408 \pm 0.001^{\mathrm{i}}$ & \\
\hline$z^{\prime}(\mathrm{mag})$ & $12.786 \pm 0.014^{\mathrm{i}}$ & \\
\hline$I_{C}(\mathrm{mag})$ & $11.65 \pm 0.13^{\mathrm{j}}$ & \\
\hline$i_{\mathrm{AB}}(\mathrm{mag})$ & & $>25.28(3 \sigma)^{\mathrm{k}}$ \\
\hline$z_{\mathrm{AB}}(\mathrm{mag})$ & & $21.75 \pm 0.07^{\mathrm{d}}$ \\
\hline$Y(\mathrm{mag})$ & & $19.4 \pm 0.05^{1}$ \\
\hline \multirow[t]{4}{*}{$J$ (mag) } & $10.211 \pm 0.022^{\mathrm{m}}$ & $18.12 \pm 0.03^{\mathrm{n}}$ \\
\hline & & $18.15 \pm 0.04(2011 / 10)$ \\
\hline & & $18.11 \pm 0.03(2011 / 12)$ \\
\hline & & $18.10 \pm 0.03(2012 / 09)$ \\
\hline$H$ (mag) & $9.598 \pm 0.022^{\mathrm{m}}$ & $17.70 \pm 0.03^{\mathrm{l}}$ \\
\hline$K_{\mathrm{S}}(\mathrm{mag})$ & $9.345 \pm 0.015^{\mathrm{m}}$ & $17.40 \pm 0.03^{1}$ \\
\hline$W 1$ (mag) & $9.241 \pm 0.022^{\circ}$ & $17.17 \pm 0.33^{\mathrm{p}}$ \\
\hline$W 2$ (mag) & $9.130 \pm 0.020^{\circ}$ & $15.41 \pm 0.22^{\mathrm{p}}$ \\
\hline W3 (mag) & $9.007 \pm 0.030^{\circ}$ & $>12.396^{\mathrm{q}}$ \\
\hline W4 (mag) & $>8.659^{q}$ & $>8.505^{\mathrm{q}}$ \\
\hline$H R 1^{\mathrm{r}}$ & $-0.17 \pm 0.24$ & \\
\hline $\begin{array}{l}\text { X-ray counts } \\
\left(\text { counts s}^{-1}\right)\end{array}$ & $(6.6 \pm 1.7) \times 10^{-2}$ & \\
\hline
\end{tabular}

Notes.

a Determined with the TiO5 index (Riaz et al. 2006).

b As a candidate member of ABDMG (see Section 3.1.1).

${ }^{c}$ Statistical distance in ABDMG from Bayesian analysis (see Section 3.1.1).

d Measured on GMOS z-band image.

e Average of PPMXL (Roeser et al. 2010), PPMX (Roeser et al. 2008), PM2000 (Ducourant et al. 2006), SDSS 9 (Ahn et al. 2012), and SUPERBLINK (Schlieder et al. 2012).

f Measured on WIRCam J-band images (2011 October and 2012 September).

g From the USNO Catalog (Monet et al. 2003).

${ }^{\text {h }}$ From SuperWASP (Norton et al. 2007).

${ }^{\mathrm{i}}$ From SDSS Photometric Catalog, DR9 (Ahn et al. 2012). Corresponds roughly to $\mathrm{AB}$ mag, except for $u^{\prime}=u_{\mathrm{AB}}+0.04$ and $z^{\prime}=z_{\mathrm{AB}}-0.02$, according to http://www.sdss.org/DR7/algorithms/fluxcal.html.

${ }^{\mathrm{j}}$ From Malo et al. (2014), considering $i^{\prime}$ from UCAC 4 catalog (APASS).

${ }^{\mathrm{k}}$ From the non detection in the second epoch GMOS $i$-band image.

${ }^{1}$ Measured in WIRCam observations.

${ }^{m}$ From 2MASS (Cutri et al. 2003).

n Average of the three epochs WIRCam observations.

${ }^{\circ}$ WISE All-Sky data Release (Cutri et al. 2012).

p Computed from WISE images (see Section 2.2.3).

q WISE 95\% confidence upper limit.

${ }^{\mathrm{r}}$ From ROSAT All-Sky Bright Source Catalogue (Voges et al. 1999).

the cross-dispersing mode with the $0^{\prime} .8$ slit, under good seeing conditions $\left(\sim 1^{\prime \prime}\right)$. The reduction was done using SpeXtool (Cushing et al. 2004; Vacca et al. 2003), and telluric absorption was corrected with the A3 spectroscopic standard star HIP 5310. 


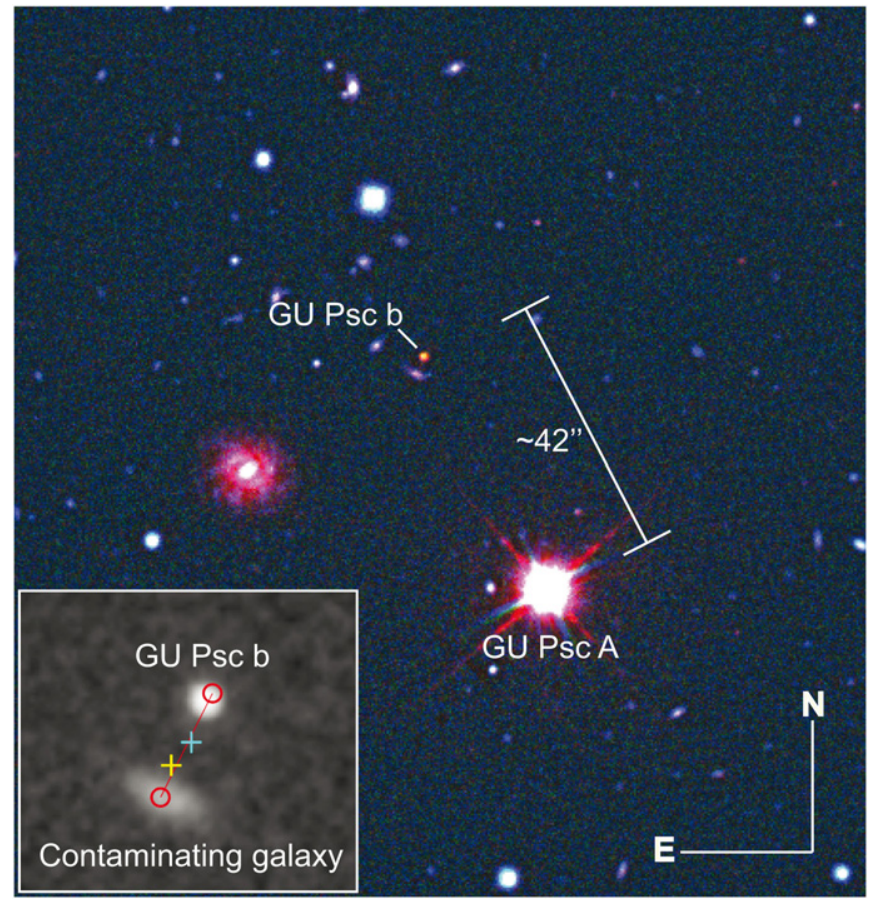

Figure 1. Main: composite Gemini-South/GMOS $i$ (blue), $z$ (green), and CFHT/WIRCam $J$ (red) image of GU Psc and its companion. As expected for a substellar object, GU Psc b is much redder in these bands than field stars and most background galaxies. Inset: close-up on GU Psc b and the galaxy located $\sim 3^{\prime \prime}$ southeast from it. The two red circles illustrate their positions at the epoch of the WISE observations, overlaid on a WIRCam $K_{\mathrm{s}}$-band image that shows both objects distinctively (a slight mismatch can be seen in the position of GU Psc $b$ since the WIRCam image was taken about 2 yr after WISE observations). The plus signs give the position of the flux barycenter in $W 1$ (yellow sign, closer to the contaminating galaxy) and in $W 2$ (blue sign, closer to GU Psc b) fluxes in WISE images; each symbol's size corresponds to the position uncertainty in that bandpass.

(A color version of this figure is available in the online journal.)

It was then flux-calibrated by adjusting the $J-H$ and $J-K_{\mathrm{s}}$ synthetic colors with the Two Micron All Sky Survey (2MASS) photometry.

\subsubsection{High-contrast Imaging}

GU Psc was observed with NICI, the high-contrast imager on Gemini-South (Ftaclas et al. 2003; Chun et al. 2008), as part of a survey to find closer-in companion (M.-E. Naud et al., in preparation). It was observed twice (2011 October 21 and 2012 September 1) in two narrowband filters around $1.6 \mu \mathrm{m}\left(\mathrm{CH}_{4} \mathrm{H}\right.$ $4 \% \mathrm{~S}$ centered at $1.578 \mu \mathrm{m}$ and the $\mathrm{CH}_{4} \mathrm{H} 4 \% \mathrm{~L}$ at $\left.1.652 \mu \mathrm{m}\right)$. Each observing sequence is composed of 35 exposures of 3 coadd $\times 20.14 \mathrm{~s}$, taken with the 0 '.32 focal plane mask. The reduction was carried using the method detailed in Artigau et al. (2008).

\subsection{The Companion, GU Psc b}

\subsubsection{Far-red Optical Photometry}

The companion was originally identified as part of a survey of young low-mass stars with Gemini-South/GMOS imaging in $i$ and $z$ bands (É. Artigau et al., in preparation). The original three $300 \mathrm{~s}$ exposures in $i$ and three $200 \mathrm{~s}$ exposures in $z$ were taken on 2011 September 22 (see Figure 1). The custom data reduction procedure included overscan and fringe subtraction and flat-field correction. Astrometry was anchored to the USNO-B1 catalog.
The images were median combined and the magnitude zero point was determined through a cross-match with the Sloan Digital Sky Survey (SDSS). Among the 91-star sample of the survey, GU Psc's companion was the only credible candidate found for separations ranging between $5^{\prime \prime}$ and $10^{\prime \prime}$ (depending on the primary's brightness) and the edge of the GMOS 5.5 field of view $(\mathrm{FoV})$. It was detected in the $z$-band image, but not in the $i$ band. Follow-up observations with the same instrument and observational setup were made on 2011 October 18 to obtain a deeper $i$-band image: five $300 \mathrm{~s} i$-band images were taken, as well as an additional $200 \mathrm{~s} z$-band image. The $z$-band photometry was consistent with that of the discovery data set, confirming that this object was not a transient or artifact. The new $i$-band imaging still did not reveal the companion but provided a $3 \sigma$ upper limit on the flux of $i>25.28$, indicating a very red $i-z$ color $(>3.53,3 \sigma)$.

\subsubsection{Near-infrared Photometry and Astrometry}

Follow-up NIR photometry was carried at the CFHT with WIRCam (Puget et al. 2004). GU Psc b was first observed in the $J$ band on 2011 October 10 for a total integration time of 14.2 minutes with single exposures of $50 \mathrm{~s}$ (see Figure 1). The target was centered on the North-East WIRCam detector and observed using a large dither pattern (15 positions or more) with the nominal $60^{\prime \prime}$ amplitude. The images were preprocessed by the IDL Interpretor of WIRCam Images ('I'iwi) ${ }^{8}$ which performs the dark subtraction, flat fielding, bad pixel masking, and sky subtraction. The final stacks were produced with SExtractor, SCAMP, and SWarp (Bertin \& Arnouts 1996; Bertin 2006; Bertin et al. 2002) and the zero point was determined using color-corrected 2MASS photometry converted to the MKO system with Leggett et al. (2006) color transformations.

NIR photometry follow-up was also made on 2011 November 5 at the $1.6 \mathrm{~m}$ telescope of the Observatoire du Mont-Mégantic, with the NIR camera CPAPIR (Artigau et al. 2004) in queue mode (Artigau et al. 2010). A set of $270 K_{\mathrm{s}}$-band images, each with two $10.1 \mathrm{~s}$ coadds were taken for a total exposure time of 91 minutes. A standard image processing (same pipeline as described in Artigau et al. 2011) was performed and yielded $K_{\mathrm{s}}=17.10 \pm 0.15$ for the object. The resulting $K_{\mathrm{s}}$ band versus Wide-field Infrared Survey Explorer (WISE) colors (see Section 2.2.3) suggested a T dwarf spectral type and prompted both additional photometric observations at the CFHT/ WIRCam and spectroscopic follow-up with Gemini-North/ GNIRS.

The second and third epoch of photometry with WIRCam in $J$ were thus acquired on 2011 December 26, 28, and 29 and on 2012 September 7 . Images in $Y, H$, and $K_{\mathrm{s}}$ were also acquired on 2012 September 7 . The observation strategy was the same as the one explained above, with total integration times of 45.8 and 30.4 minutes, respectively, in the two $J$-band epochs and 30.4 , 19.0, and 8.3 minutes for the $Y, H$, and $K_{\mathrm{s}}$ stacks, respectively. Single exposures were $150 \mathrm{~s}, 50 \mathrm{~s}, 15 \mathrm{~s}$, and $25 \mathrm{~s}$ for $Y, J, H$, and $K_{\mathrm{s}}$, respectively. The $Y$-band zero point was determined through the observation of a spectrophotometric standard. The 2011 October and 2012 September $J$-band images allowed precise multi-epoch astrometric measurements: a linear astrometric solution was determined for each based on the 2MASS point source catalog (Cutri et al. 2003).

\footnotetext{
8 http://www.cfht.hawaii.edu/Instruments/Imaging/WIRCam/ IiwiVersion1Doc.html
} 

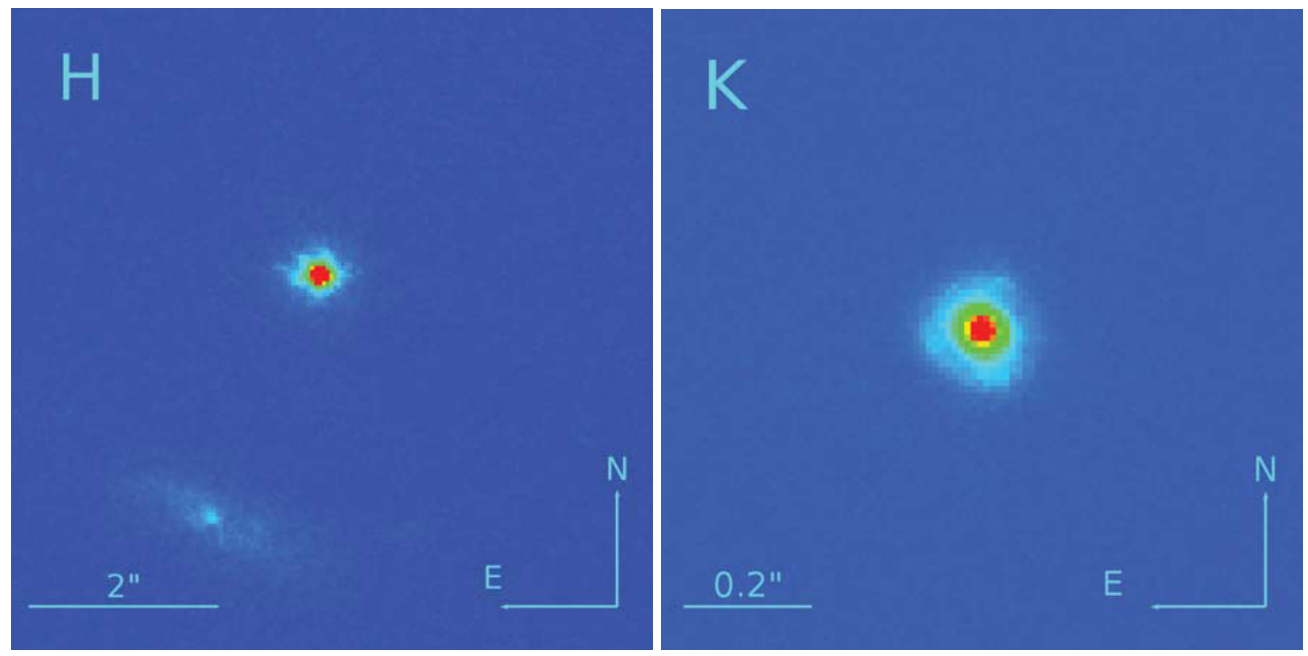

Figure 2. LGS AO $H$-and $K$-band images of the GU Psc b. Left: the $H$-band image shown is 6. 8 wide, the galaxy present in the inset of Figure 1 can be seen in the lower left corner. Right: the $K$-band image shown here is $\sim 1^{\prime \prime} .7$ on a side. There is no other point source detected in the FOV $\left(10^{\prime \prime} .2 \times 10^{\prime \prime} 2\right)$.

(A color version of this figure is available in the online journal.)

\subsubsection{WISE Photometry}

In the WISE All-Sky Source Catalog ${ }^{9}$ (Cutri et al. 2012), there is a source detected at the position of the companion with $W 1=15.818 \pm 0.064$ and $W 2=15.039 \pm 0.120$. However, the WIRCam, GMOS, and CPAPIR images also show a faint extended object, most likely an edge-on spiral galaxy, $\sim 3^{\prime \prime}$ southeast of GU Psc b. The inset of Figure 1 shows the position of GU Psc b and of the galaxy at the epoch of the WISE measurements (red circles), overlaid on the WIRCam $K_{\mathrm{s}}$-band image. The position of GU Psc b was deduced from WIRCam images and the proper motion in Table 1 . The yellow and cyan plus signs show the WISE $W 1$ and $W 2$ fluxes barycenters, respectively. They both fall within $1 \sigma$ on the line joining GU Psc $\mathrm{b}$ and the interloping galaxy, which confirms that the WISE photometry is a blend of the two objects. The position along the line allows one to derive the relative contribution of each object to the blended flux of the catalog. The galaxy contributes $71 \% \pm 10 \%$ of the $W 1$ flux and $46 \% \pm 10 \%$ of the $W 2$ flux. The resulting photometry for GU Psc b, listed in Table 1, is overall consistent with mid-T photometry for field objects (e.g., Kirkpatrick et al. 2011; Dupuy \& Liu 2012).

\subsubsection{Near-infrared Spectroscopy}

The spectrograph GNIRS on Gemini-North was used in its cross-dispersed mode to obtain an NIR 0.9-2.4 $\mu \mathrm{m}$ spectrum with a resolving power of $R \sim 800$. We used the 0' 675 slit, the short $\left(0\right.$ ' 15 pixel $\left.^{-1}\right)$ camera and the $31.71 \mathrm{~mm}^{-1}$ grating. The target observations, acquired on 2012 November 12, were followed by the observation of an A0 spectroscopic standard star (HIP13917) to calibrate the flux and correct for telluric absorption lines. A total of $1.2 \mathrm{hr}$ of observation was taken, subdivided in five $180 \mathrm{~s}$ exposures. The reduction was carried with the pipeline presented in Delorme et al. (2008) and Albert et al. (2011). Wavelength calibration was made using bright $\mathrm{OH}$ sky lines (Rousselot et al. 2000). To perform the flux calibration, the spectrum was integrated over the WIRCam $Y, J, H$, and $K_{\mathrm{s}}$ bandpasses, respectively. ${ }^{10}$ For each filter, we then evaluated the

\footnotetext{
9 Available at http://wise2.ipac.caltech.edu/docs/release/allsky/.

10 Using the transmission curves available at

http://www.cfht.hawaii.edu/Instruments/Filters/wircam.html.
}

factor by which one must multiply the integrated flux to get the WIRCam photometric measurements. A linear fit of the factors was used to rectify every wavelength of the spectrum. The calibrated spectrum is shown in Figures 6, 8, and 9, along with photometric points outside the range covered by the spectrum $(z, W 1$, and $W 2)$.

\subsubsection{High-resolution Near-infrared Imaging}

On 2013 November 19, we used the sodium Laser Guide Star (LGS) AO system of the Keck II Telescope (Wizinowich et al. 2006; van Dam et al. 2006), located on the summit of Mauna Kea, in Hawaii, to verify if the companion is a resolved binary. The LGS constitutes the reference wavefront for the AO correction, and the tip-tilt was monitored using the close (26".4 away) star SDSS J011237.05+170456.9 $\left(r_{\mathrm{AB}}=15.742 \pm 0.004\right.$; SDSS DR 9; Ahn et al. 2012). We used the near-infrared camera NIRC2 in its narrow mode $\left(\mathrm{FoV} 10^{\prime \prime} \times 10^{\prime \prime}\right.$, pixel scale of 9.942 mas pixel ${ }^{-1}$ ) for the $K$-band images and in its wide mode $\left(\mathrm{FoV} 40^{\prime \prime} \times 40^{\prime \prime}\right.$, pixel scale of $39.686 \mathrm{mas}^{\mathrm{p} i x e l^{-1}}$ ) for the $H$-band images. We obtained 12 images of two coadditions of $60 \mathrm{~s}$ in $K$ band, for a total integration time of 24 minutes, and nine images of four coadditions of $30 \mathrm{~s}$ in $H$ band, for a total integration time of 18 minutes. The images were obtained using a three-point dither pattern that avoided the noisy quadrant in the lower-left portion of the array. The positional offset between images varied between $1^{\prime \prime} .5$ and $3^{\prime \prime}$. Conditions were photometric during the observation. Standard reduction techniques were used: images were divided by the dome flat and a median sky image constructed from all the observations of the night was subtracted. Then every image was shifted and stacked to produce a final image in each band (see Figure 2).

\section{RESULTS}

\subsection{Physical Properties of the Host Star}

\subsubsection{GU Psc: A Young Low-mass Star Candidate Member of the AB Doradus Moving Group}

GU Psc was originally identified as a highly probable M dwarf by Zickgraf et al. (2003). Then, Riaz et al. (2006) identified it as an M3 \pm 0.5 using the TiO5 index (Reid et al. 1995) and obtained a visible spectrum that allowed the measurement of its 
Table 2

Radial Velocity and Projected Rotational Velocity of GU Psc A

\begin{tabular}{lllcc}
\hline \hline & \multicolumn{1}{c}{ Date } & \multicolumn{1}{c}{$\begin{array}{c}v_{\text {rad }} \\
\left(\mathrm{km} \mathrm{s}^{-1}\right)\end{array}$} & $\begin{array}{c}v \sin i \\
\left(\mathrm{~km} \mathrm{~s}^{-1}\right)\end{array}$ & Note \\
\hline Measured & 2010 Nov 19 & $0.4 \pm 1.2$ & $25.4 \pm 2.6$ & $\mathrm{a}$ \\
& 2012 Jul 11 & $-1.8 \pm 0.7$ & $23.1 \pm 2.3$ & $\mathrm{~b}$ \\
& 2013 Jul 21 & $-1.7 \pm 0.7$ & $24.1 \pm 2.3$ & $\mathrm{~b}$ \\
& 2012 Jan 6 & $-1.6 \pm 0.3$ & $22.5 \pm 0.9$ & $\mathrm{c}$ \\
& Average & $-1.6 \pm 0.4$ & $23.0 \pm 1.4$ & $\mathrm{~d}$ \\
Predicted & & $-1.5 \pm 1.9 \mathrm{e}$ & & \\
\hline
\end{tabular}

Notes.

a Gemini-S/PHOENIX NIR spectroscopy.

b VLT/CRIRES NIR spectroscopy.

c ESPaDOnS/CFHT optical spectroscopy.

d Weighted average of the four measures.

e Bayesian analysis (Malo et al. 2013).

$\mathrm{H} \alpha$ emission line equivalent width (EW), which is a proxy for its chromospheric activity, and thus youth.

Through Bayesian inference, GU Psc was then identified by Malo et al. (2013) as a highly probable (98\%) member of the ABDMG. The Bayesian analysis makes use of a priori knowledge of known associations (Galactic position, space velocity, and $I_{C}-J$ absolute photometry) and compares these properties with observables of a given candidate (sky position, proper motion, and $I_{C}$ and $J$ apparent magnitudes). The analysis gives as an output the membership probability of the star for every association considered as well as the probability that it is a field star unrelated to these associations. It also gives the most probable radial velocity the candidate should have if it were a true member of a given association (accurate to a few $\mathrm{km} \mathrm{s}^{-1}$ ) and the most probable statistical distance $d_{s}$ it would have. Malo et al. (2013) showed that this statistical distance agrees with the trigonometric distance within $\sim 10 \%$ for bona fide members of the associations. For GU Psc, using the average proper motion shown in Table 1 , they derived $d_{s}=$ $48 \pm 5 \mathrm{pc}$, which is the value we adopt for the distance hereafter and predicted a $v_{\text {rad }}=-1.5 \pm 1.9 \mathrm{~km} \mathrm{~s}^{-1}$. Using any of the proper motion measurements available in the literature does not change these results significantly. As part of a comprehensive radial velocity follow-up program (Malo et al. 2014), multiepoch radial velocity measurements of GU Psc through NIR and optical spectroscopy were secured.

The results, summarized in Table 2, yield a weighted average radial velocity of $\left\langle v_{\mathrm{rad}}\right\rangle=-1.6 \pm 0.4 \mathrm{~km} \mathrm{~s}^{-1}$, in excellent agreement with the predicted radial velocity for membership in ABDMG. Adding the radial velocity observable to the Bayesian analysis yields an increased membership probability of $99.9 \%$ for ABDMG. These observations also show that GU Psc is a relatively fast rotator with a $v \sin i$ of $23 \mathrm{~km} \mathrm{~s}^{-1}$.

Note that, as mentioned in Gagné et al. (2014), the probabilities mentioned here should not be interpreted as absolute. Even though any given star is a priori less likely to belong to a given association than to the field (there are much less stars in the association than in the field), the precise values of these prior probabilities are very uncertain so all hypotheses are considered as equally likely in the analysis of Malo et al. (2013). They estimate that for the candidates of ABDMG with a membership probability over $90 \%$, the contamination rate (false positive) is about $14 \%$.

We also used the analysis of Gagné et al. (2014) to evaluate GU Psc's membership. This analysis differs from that of Malo et al. (2013) in two major aspects. First, it uses a different method to outline the three-dimensional regions covered by the bona fide members of an association in the Galactic position space $X, Y, Z$ and in the Galactic velocity space $U, V, W$. While Malo et al. (2013) uses ellipsoids with the three axes aligned on the Galactic coordinate system, Gagné et al. (2014) use a more realistic approach where the ellipsoids can be aligned in any direction. Second, Gagné et al. (2014) uses the knowledge of the distance of known members of a given association as an additional prior on the plausible distance that a candidate can have. In agreement with Malo et al. (2013), this analysis points toward a membership in ABDMG for GU Psc, albeit with a smaller probability (88\%). It finds a compatible statistical distance of $47 \pm 5 \mathrm{pc}$ and a very similar predicted radial velocity $\left(-1.8 \pm 2.0 \mathrm{~km} \mathrm{~s}^{-1}\right)$. It also yields a non-negligible probability of $12 \%$ for the membership in the younger (12-22 Myr) $\beta$ Pictoris Moving Group ( $\beta$ PMG), associated with a smaller statistical distance, $d_{s}=32 \pm 3 \mathrm{pc}$.

Thus, both analyses suggest that GU Psc is a member of a young association, either ABDMG or $\beta \mathrm{PMG}$, with a much higher probability for the former.

\subsubsection{The Age of the AB Doradus Moving Group}

The age of ABDMG, first identified as a moving group by Zuckerman et al. (2004), is subject to debates. The comparative analysis of ABDMG and the open cluster IC 2391 in an $M_{V}$ versus $V-K$ color-magnitude diagram led Luhman et al. (2005) to derive an age between 75 and $150 \mathrm{Myr}$, roughly coeval with the Pleiades (for which they adopted an age of 100-125 Myr). Using an $M_{I}$ versus $V-I$ diagram and the lithium EW, Lopez Santiago et al. (2006) formulated the hypothesis that the group could be composed of two subgroups: one younger (30-50 Myr) and one older (80-120 Myr). The Li EW was also used in two other studies to deduce a lower limit on the age of $45 \mathrm{Myr}$ (Mentuch et al. 2008) and an age of 70 Myr (da Silva et al. 2009). Recently, Barenfeld et al. (2013) studied the kinematics and the abundance of 10 different elements in 10 members of the "stream" (i.e., stars that are not among the nine stars considered as the "nucleus" by Zuckerman et al. 2004) and pointed out that many stars traditionally associated with ABDMG do not have a similar chemical composition and/or were not likely formed at the same position as the ABDMG nucleus. They concluded that the remaining members are at least 110 Myr, based on the fact that the group still has zero-age main sequence $\mathrm{K}$ stars members. Considering all these studies, we adopt a conservative age of $100 \pm 30 \mathrm{Myr}$ for ABDMG as a whole.

\subsubsection{Youth Indicators}

To better constrain the age of GU Psc, we consider here other age indicators. Table 3 summarizes all the information on the age of the GU Psc system.

Over time, the coronal activity that is induced by magnetic field is reduced, which causes the X-ray emission to decrease (Preibisch \& Feigelson 2005). Using GU Psc's X-ray count rate and hardness ratio (HR1) measured by ROSAT (Voges et al. 1999; see Table 1) in the relation given in Schmitt et al. (1995) yields a value of $4.90 \times 10^{-13} \mathrm{erg} \mathrm{s}^{-1} \mathrm{~cm}^{-2}$ for the $X$-ray flux, thus an X-ray luminosity of $\log L_{X}=29.1 \pm 0.3 \mathrm{erg} \mathrm{s}^{-1}$ at $d_{s}=$ $48 \pm 5 \mathrm{pc}$. This $X$-ray luminosity is very similar to that of single low-mass ABDMG members; if we use a similar procedure to evaluate the $\log L_{X}$ of the six bona fide $\mathrm{M}$ dwarfs members listed in Malo et al. (2013), we obtain $\log L_{X}=29.03 \mathrm{erg} \mathrm{s}^{-1}$, with a dispersion of 0.07 dex. The X-ray luminosity of GU 
Table 3

Age Estimate of the GU Psc System

\begin{tabular}{lc}
\hline \hline Method & Range of Age Allowed \\
\hline $\begin{array}{l}\text { Bayesian analysis (kinematic } \\
\text { and photometry) }\end{array}$ & ABDMG candidate member \\
& $(100 \pm 30 \mathrm{Myr})$ \\
\hline X-ray emission & Similar to ABDMG members \\
H $\alpha$ emission EW & $<2 \pm 0.5 \mathrm{Gyr}$ \\
H $\alpha$ emission W10\% & $>10 \mathrm{Myr}$ \\
No infrared excess & $>10 \mathrm{Myr}$ \\
Li absorption absent & $>22 \mathrm{Myr}$ \\
Rotation period & If $M_{\star}>0.35 M_{\odot},<650 \mathrm{Myr}$ \\
& If $M_{\star}<0.35 M_{\odot}$, no constraint \\
\hline Adopted & $100 \pm 30 \mathrm{Myr}$ \\
\hline
\end{tabular}

Psc is also consistent with that of other candidate members of ABDMG, such as the M3.5 star J01225093-2439505 (hereafter $2 \mathrm{M} 0122$ ) that has $\log L_{X}=28.7 \pm 0.2 \mathrm{erg} \mathrm{s}^{-1}$ (Bowler et al. 2013), or the M2 star J235133.3+312720, that has a $\log L_{X}=$ $29.3 \pm 0.2 \mathrm{erg} \mathrm{s}^{-1}$ (Bowler et al. 2012). GU Psc's X-ray luminosity is however significantly higher than that of field stars of similar mass. For example, the $\log L_{X}$ of the 42 single field $\mathrm{M}$ dwarfs listed in Malo et al. (2014) has a mean of $\log L_{X}=27.6 \mathrm{erg} \mathrm{s}^{-1}$ with a dispersion of $0.5 \mathrm{dex}$. At the statistical distance for the $\beta$ PMG given by Gagné et al. (2014) analysis $\left(d_{s}=32 \pm 3 \mathrm{pc}\right)$, GU Psc's X-ray luminosity would be $\log L_{X}=28.8 \pm 0.3 \mathrm{erg} \mathrm{s}^{-1}$, which is also not consistent with that of $\beta$ PMG members: the mean value computed for the nine single bona fide $\mathrm{M}$ dwarfs of the $\beta$ PMG in Malo et al. (2013) is $\log L_{X}=29.63 \mathrm{erg} \mathrm{s}^{-1}$, with a dispersion of $0.16 \mathrm{dex}$. The $\mathrm{X}$-ray activity thus favors an ABPMG membership and not a $\beta$ PMG membership.

The reduction of the magnetic activity occurring as the star evolves is also traceable by the diminution of the $\mathrm{H} \alpha$ emission line at $6562.8 \AA$. In our visible spectrum (Figure 3(a)), we measure $\mathrm{EW}_{\mathrm{H} \alpha}=-3.96 \AA$. According to West et al. (2008), the activity lifetime of an M3 is $2 \pm 0.5 \mathrm{Gyr}$, thus the presence of $\mathrm{H} \alpha$ in emission implies that GU Psc is likely younger than this. Also, according to the criteria developed in White \& Basri (2003), the $10 \%$ width of the same $\mathrm{H} \alpha$ emission line (W10\% = $125 \mathrm{~km} \mathrm{~s}^{-1}$ ) is consistent with a star that is non accreting (W10\% $<270 \mathrm{~km} \mathrm{~s}^{-1}$ ), thus older than $\sim 10 \mathrm{Myr}$ (Barrado y Navascués \& Martín 2003). This lower limit is also consistent with the fact that no disk is seen in the form of a mid-infrared excess. Indeed, when the $J, H, K_{\mathrm{s}}$ bands are fitted with a model spectrum (BT-Settl AGSS2009; Allard et al. 2012), the WISE photometry in the four bands falls directly on the model spectral energy distribution (SED). These three indicators are consistent with a membership in either ABDMG or $\beta$ PMG.

The absence of the lithium absorption line at $6708 \AA$ in the optical spectrum of GU Psc $\left(\mathrm{EW}_{\mathrm{Li}} \lesssim 18 \mathrm{m \AA}, 3 \sigma\right.$; see Figure 3(b)) also yields a minimum age for GU Psc. Indeed, the early $\mathrm{M}$ members of ABDMG show lithium measurements compatible with this upper limit (Mentuch et al. 2008; Yee \& Jensen 2010). Our upper limit is also compatible with the wide range of lithium absorption that the low-mass stars show at the younger age (12-22 Myr) of $\beta \mathrm{PMG}$, which varies from an upper limit of a few tenths of $\mathrm{m} \AA$ up to $\sim 500 \mathrm{~m} \AA$ (Mentuch et al. 2008; Binks \& Jeffries 2014). Indeed, Figure 5 of Mentuch et al. (2008) shows that the youngest association for which early $\mathrm{M}$ stars show little or no lithium is $\beta \mathrm{PMG}$; there is no early $\mathrm{M}$ star with $\mathrm{EW}_{\mathrm{Li}} \lesssim 350 \AA$ in the TW Hydrae Association (TWA) nor in the $\eta$ Chamaelontis Cluster, which are both $\lesssim 20 \mathrm{Myr}$

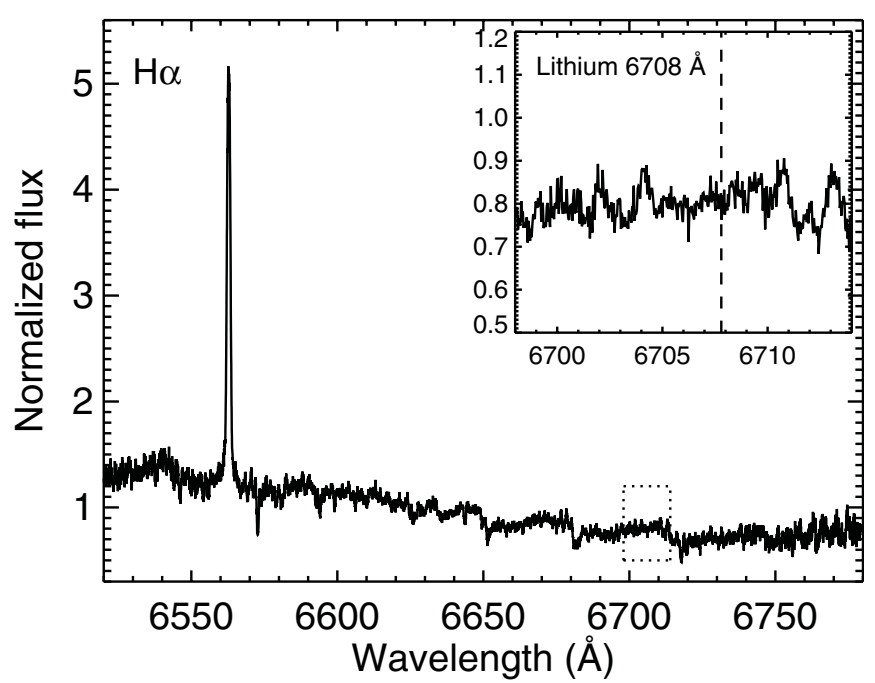

Figure 3. Host star ESPaDOnS optical spectrum between $6520 \AA$ and $6780 \AA$. The $\mathrm{H} \alpha$ emission line at $6562.8 \AA$ is clearly seen. The equivalent width of the band is $-3.96 \AA$. The $6708 \AA$ lithium absorption line is however not detected, we measure a $3 \sigma$ upper limit of $\mathrm{EW}_{\mathrm{Li}} \lesssim 18 \mathrm{~m} \AA$.

(e.g., Fernández et al. 2008). We can thus estimate that GU Psc is likely older than $\sim 22$ Myr.

For an object with a mass above the fully convective limit ( $\gtrsim 0.35 M_{\odot}$, Chabrier \& Baraffe 1997), the rotation rate increases as the star contracts toward the main sequence, reaches a plateau at an age comparable to that of ABDMG, and then slows down due to various interactions (Sills et al. 2000). The rotation period can thus help to constrain the age. According to the SuperWASP photometric survey, GU Psc is a variable star with a $1.0362 \pm 0.0005$ day period (Norton et al. 2007). That could be indicative of a relatively fast rotator, which is also suggested by its relatively large $v \sin i$ of $23 \mathrm{~km} \mathrm{~s}^{-1}$. If GU Psc is not fully convective $\left(>0.35 M_{\odot}\right)$, the $\sim 1$ day rotation period suggests an upper limit on the age of $\sim 650 \mathrm{Myr}$ (see Figure 12 in Irwin et al. 2011). With the $I_{C}-J$ listed in Table 1 and the age of ABDMG $(100 \pm 30 \mathrm{Myr})$, GU Psc's mass $M_{\star}$ is estimated to be between 0.30 and $0.35 M_{\odot}$ (using the models of Baraffe et al. 1998), which is very close to the limit for a star to be fully convective. Without a parallax, it is challenging to determine whether or not GU Psc has a fully convective structure. If GU Psc is fully convective, the upper limit of the age we can set using the rotation period is much greater, since the spin-down time of fully convective objects is very long ( $>5 \mathrm{Gyr}$; Irwin et al. 2011).

It is interesting that HIP 17695, the only single bona fide member of ABDMG with a spectral type similar to GU Psc (M2.5; Malo et al. 2013), has a $v \sin i\left(18 \mathrm{~km} \mathrm{~s}^{-1}\right.$; da Silva et al. 2009) and rotation period ( $P_{\mathrm{rot}}=3.87$ days; Messina et al. 2010) that are close to those of GU Psc. This object is probably at the low-age end of ABDMG ( $70 \mathrm{Myr})$, given its X-ray luminosity, $\mathrm{H} \alpha$ and Li EW (Zuckerman et al. 2004).

Considering that the Bayesian analysis favors a membership in $\mathrm{ABDMG}$, and that other youth indicators suggest an age consistent with that association, we adopt hereinafter the age of ABDMG (100 $\pm 30 \mathrm{Myr})$ and the associated statistical distance $\left(d_{s}=48 \pm 5 \mathrm{pc}\right)$ for GU Psc's system.

\subsubsection{Metallicity}

We evaluated the metallicity of GU Psc using two metallicity calibrations developed recently, specifically for $\mathbf{M}$ dwarfs. 


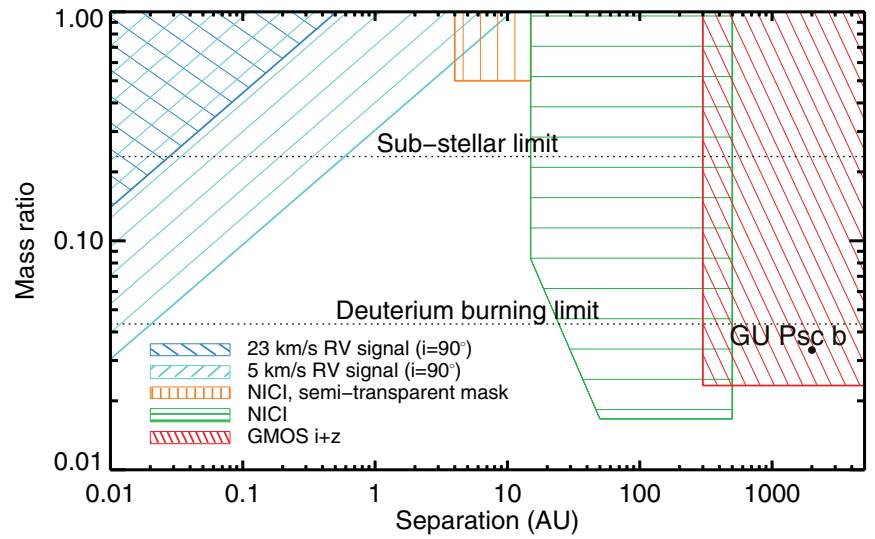

Figure 4. Constraints on the presence of another companion around GU Psc A. The shape of the rotation-broadened line profile in the CRIRES and ESPADONS spectra exclude the blue region $\left(v \sin i>23 \mathrm{~km} \mathrm{~s}^{-1}\right)$. The multi-epoch radial velocity measurements are stable to within $1 \mathrm{~km} \mathrm{~s}^{-1}$ (see Table 2) and match that expected for the ABDMG along the line of sight to within $1 \mathrm{~km} \mathrm{~s}^{-1}$. This conservatively rules out SB1 binaries with a radial velocity semi-amplitude $v \sin i>5 \mathrm{~km} \mathrm{~s}^{-1}$ (cyan area). The spectroscopic constraints are shown for systems with inclinations close to edge-on $\left(i \sim 90^{\circ}\right)$. At separations between a few AUs and a few hundred AUs, NICI high-contrast imaging allows ruling out companions, down to the planetary-mass regime for the largest separations (green and orange area). At separations greater than a few hundred AUs, GMOS imaging eliminates the possibility of another companion down to planetary-mass regime (red region).

(A color version of this figure is available in the online journal.)

One must bear in mind however that these calibrations were developed for field stars and are not necessarily appropriate for young stars. Newton et al. (2014) calibration is based on the $\mathrm{Na}$ line at $2.2 \mu \mathrm{m}$. For GU Psc, we obtain $[\mathrm{Fe} / \mathrm{H}]=$ $+0.10 \pm 0.13$. Mann et al. (2013) improves previous calibrations (notably Terrien et al. 2012 and Rojas-Ayala et al. 2012) and presents metallicity calibrations based on various features in optical and NIR. We obtained $[\mathrm{Fe} / \mathrm{H}]_{H}=-0.14 \pm 0.09$ with the $H$-band calibration and $[\mathrm{Fe} / \mathrm{H}]_{K}=0.04 \pm 0.08$ with the $K$-band calibration. ${ }^{11}$ While the value derived using the $H$ band is slightly lower than the others, the ones derived with Mann et al. (2013) and Newton et al. (2014) using $K$ band are consistent, within uncertainties, with each other and with the one derived by Barenfeld et al. (2013) for 10 ABDMG members, $[\mathrm{Fe} / \mathrm{H}]=$ $+0.02 \pm 0.02$.

\subsubsection{Constraints on Multiplicity of the Host Star}

The high-contrast imaging and high-resolution spectroscopy observations we made on the primary provide strong constraints on the mass ratio and separation of a possible planetary-mass, brown dwarf, or stellar companion (see Figure 4).

First, high-resolution spectroscopy shows a single-line profile. Considering the measured projected rotational velocity of GU Psc $\left(v \sin i \sim 23 \mathrm{~km} \mathrm{~s}^{-1}\right.$; Table 2), this excludes a double-line binary (SB2) with components showing $\Delta v \sin i>$ $23 \mathrm{~km} \mathrm{~s}^{-1}$. Second, the multi-epoch radial velocity measurements obtained with ESPaDOnS and CRIRES (Table 2) are stable at the $\mathrm{km} \mathrm{s}^{-1}$ level and consistent with the value predicted for an ABDMG member in GU Psc's line of sight within $<1 \mathrm{~km} \mathrm{~s}^{-1}$, which excludes many cases of single-line binary (SB1). We adopt a conservative upper limit on GU Psc's radial velocity semi-amplitude of $v \sin i<5 \mathrm{~km} \mathrm{~s}^{-1}$, since a larger discrepancy between the measured radial velocity and the one predicted for GU Psc in ABDMG would

\footnotetext{
11 See the IDL program available online at

http://ifa.hawaii.edu/ amann/programs/am_getmetal.pro.
}

correspond to a $3 \sigma$ outlier. Note however that for both spectroscopic constraints $\left(v \sin i>23 \mathrm{~km} \mathrm{~s}^{-1}\right.$ and $v \sin i>$ $5 \mathrm{~km} \mathrm{~s}^{-1}$ ), a near-equal luminosity binary system would not be detected for nearly pole-on geometries. In the case of the second constraint $\left(v \sin i>5 \mathrm{~km} \mathrm{~s}^{-1}\right)$, a companion with the same luminosity as GU Psc would not be detected either. The blue and cyan regions of Figure 4 show the mass ratio and separation ranges excluded by these two constraints, respectively, for an orbit orientation of $i \sim 90^{\circ}$.

At greater separations, no companions (other than GU Psc b) were found through imaging observations with NICI or GMOS. Inside NICI's semi-transparent mask, which absorbs $\sim 6$ mag in the central 0.32 radius region ( $15 \mathrm{AU}$ at $d_{s}=48 \mathrm{pc}$ ), no object is seen at separations greater than one FWHM of the point-spread function $\left(0{ }^{\prime} 08\right.$, or $\left.4 \mathrm{AU}\right)$, down to a flux ratio of 4 (1.5 mag). The NICI data were taken with the $4 \% \mathrm{CH}_{4}$ on and off filters within the $H$ band; a companion 1.5 mag fainter than GU Psc would have $M_{H}=9.3$ which corresponds to a $\sim \mathrm{M} 6$ spectral type (Dupuy \& Liu 2012) and a temperature $400 \mathrm{~K}$ cooler than GU Psc (i.e., respectively $\sim 3300 \mathrm{~K}$ and $\sim 2900 \mathrm{~K}$, the difference being more accurate than the absolute values, see Figure 5 in Rajpurohit et al. 2013). Using the BT-Settl model (AGSS2009; Allard et al. 2012) at an age of $100 \mathrm{Myr}$, a difference of $400 \mathrm{~K}$ for a primary star in the $3000-3500 \mathrm{~K}$ range leads to a maximal mass ratio of $32 \%-54 \%$. The orange region of Figure 4 shows the excluded region from this observation, adopting $50 \%$ as a conservative upper limit on the mass ratio. NICI imaging beyond the edge of the mask shows no background companion out to a separation of $9^{\prime \prime}(430 \mathrm{AU})$ at a $5 \sigma$ contrast of $\Delta H \sim 12$, yielding an upper limit of $M_{H} \sim 18$, or a mass limit of $\sim 5 M_{\text {Jup }}$ (green region of Figure 4). The GMOS imaging has a $5 \sigma z$-band limit at 23.2, which translates to a mass limit of $\sim 7 M_{\text {Jup }}$ and, thus, a mass ratio of $2.3 \%$ (red region of Figure 4 ).

Malo et al. (2013) mentioned the possibility that GU Psc might be a binary star. Indeed, the absolute magnitude GU Psc assuming $d_{s}=48 \mathrm{pc}, M_{J}=6.80$, is about 0.78 mag brighter than the one predicted for a single ABDMG member with GU Psc's color $\left(I_{C}-J=1.44\right.$; see the $M_{J}$ versus $I_{C}-J$ color-magnitude diagram on Figure 3 in Malo et al. 2013). The magnitude dispersion along the ABDMG empirical sequence for that $I_{C}-J(\sim 0.5 \mathrm{mag}$; Malo et al. 2013) is important. A limited number of bona fide low-mass members are known in this association and there is probably an intrinsic age spread among the members. Thus, this over luminosity may not be significant. If it is, it could be due to an unseen companion, but it could also be attributed to other factors (for example, to an important chromospheric activity; Riedel et al. 2011).

In conclusion, the various data sets we obtained pose stringent constraints on the presence of another companion around GU Psc. The near-equal luminosity binary scenario can be virtually excluded at all separations, unless a rather unlikely geometry is invoked, such as a near pole-on geometries for SB1 or SB2 cases or an alignment of a companion behind the star at the time of the high-resolution imaging observations. Besides, there is still a possibility of a stellar companion with a maximum mass of about half that of GU Psc's between 1 and $10 \mathrm{AU}$ or of a brown dwarf companion inward of $\sim 10 \mathrm{AU}$.

\subsection{Physical Properties of the Companion}

\subsubsection{Proper Motion}

Figure 5 shows the proper motion of GU Psc and of GU Psc b. For the primary, there are several proper motion measurements 


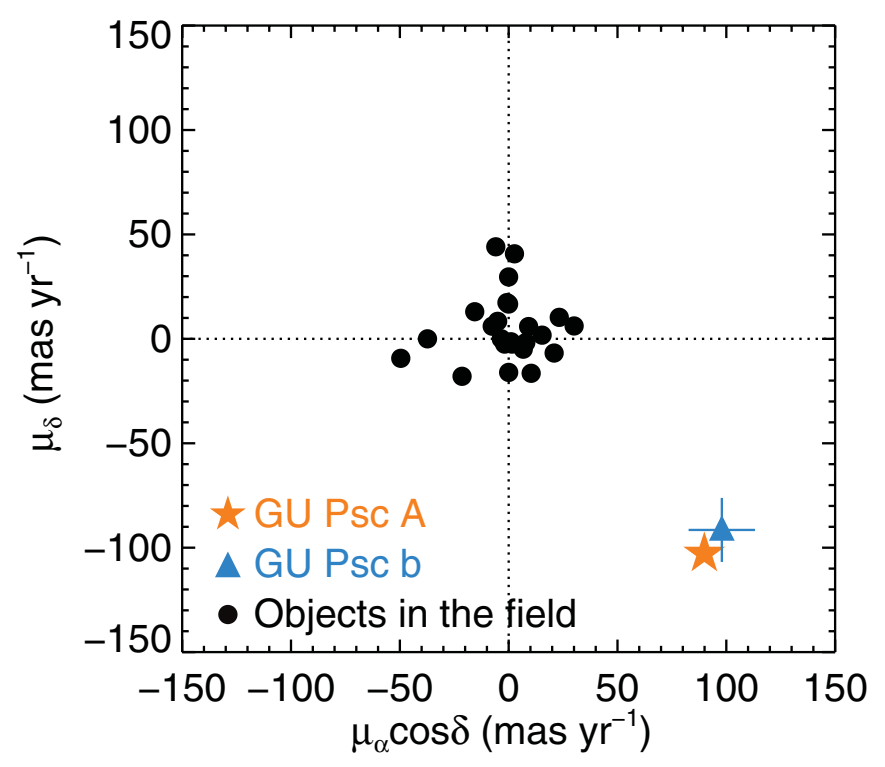

Figure 5. Proper motion in declination and right ascension for GU Psc A, GU Psc b, and stars in the field computed with the 2011 October and 2012 September $J$-band WIRCam images. The primary is saturated on these images so a weighted average of the proper motions found in the literature is shown. The uncertainty on GU Psc A proper motion is smaller than the plot symbol.

(A color version of this figure is available in the online journal.)

reported in the literature (Roeser et al. 2010, 2008; Ducourant et al. 2006; Ahn et al. 2012; Schlieder et al. 2012). Since these measurements are not independent, we adopt the mean value and use the average uncertainties as the error of the resulting

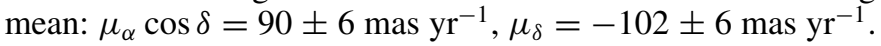
For the companion, we used the two $J$-band epochs taken at CFHT/WIRCam in 2011 October and 2012 September (11 months apart) and found $\mu_{\alpha} \cos \delta=98 \pm 15 \mathrm{mas} \mathrm{yr}^{-1}, \mu_{\delta}=$

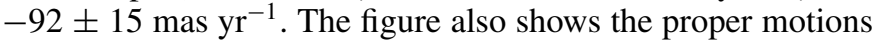
of stars in the WIRCam image field, computed like that of GU Psc b. The companion proper motion is clearly consistent with that of the star and inconsistent with that of the other stars in the field, thus confirming it is gravitationally bound.

This conclusion is strengthened by the fact that the probability of finding an unassociated $\mathrm{T}$ dwarf with this proper motion in the vicinity of a young $\mathrm{M}$ dwarf is very low. To assess this probability, we first consider only proper motion. Using the 64 T dwarfs in the Dupuy \& Liu (2012) sample (Table 9) that have a parallax and proper motion with errors smaller than $10 \%$, we compute a median sky plane velocity, $35 \mathrm{~km} \mathrm{~s}^{-1}$, which we assume to be typical of T dwarfs. Assuming an isotropic random Gaussian distribution of velocities, this velocity corresponds to a Gaussian dispersion per coordinate of $30 \mathrm{~km} \mathrm{~s}^{-1}$, which

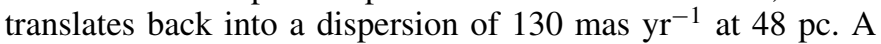
numerical integration of a two-dimensional Gaussian shows that only $\sim 1.5 \%$ of random T dwarfs would match GU Psc's proper motion at the $<2 \sigma$ level. Then, we consider the sky position. The local density of T0-T5.5 dwarfs is $1.4_{-0.2}^{+0.3} \times 10^{-3} \mathrm{pc}^{-3}$ (Reylé et al. 2010), there are therefore $\sim 730$ early $\mathrm{T}$ dwarfs within $50 \mathrm{pc}$ of the Sun. Our survey sampled a near-circular field with a $5^{\prime \prime} .5$ diameter around 91 stars, covering a total area of $0.6 \mathrm{deg}^{2}$ or $1.5 \times 10^{-5}$ of the entire sky. There is thus only a $\sim 1 \%$ chance of finding a random single field early-T dwarf with properties comparable to GU Psc b within a GMOS FoV of one survey stars. The combined likelihood of a false positive match in both proper motion and position is therefore of the order of
$2 \times 10^{-4}$, and it is likely to be much lower as GU Psc and GU Psc $b$ both display signs of youth.

\subsubsection{Constraints on Multiplicity of the Companion}

The $H$ - and $K$-band observations of the companion made with LGS AO at Keck show only one object. The companion is thus not a resolved binary. The $K$-band image excludes, at the $>5 \sigma$ level, any second object that would have a $\Delta K \lesssim 4$ for separations between 0.08 and $10^{\prime \prime}(\sim 4-480 \mathrm{AU}$ at $48 \mathrm{pc})$. Using $M_{K} \sim 14$ for GU Psc b, this $\Delta K$ corresponds approximately to a T8 or later, according to the polynomial relations given in Dupuy \& Liu (2012). For smaller separations, the $K$-band observations exclude a companion brighter than a typical T7.5 down to a separation of $0{ }^{\prime} .04$ ( $\left.~ 2 \mathrm{AU}\right)$. The wider $H$-band image does not show any object (besides a few galaxies) down to a $\Delta H$ of $\sim 3.6$, in a radius between 0 '.7 and the width of the field, $40^{\prime \prime}$ ( $\sim 30-1900 \mathrm{AU}$ at $48 \mathrm{AU})$. Considering $M_{H}=14.3$ for GU Psc $\mathrm{b}$, this excludes a companion earlier than $\sim \mathrm{T} 7.5$ in that region.

GU Psc b could still be a very tight binary object, but these observations largely exclude a T5-T6 companion in a wide range of distances and down to the typical separations of T dwarfs binaries (e.g., SDSS J153417.05+161546.1, a T1.5+T5.5 with a separation of 3.96 AU; Liu et al. 2006, SDSS J102109.69-030420.1, J1021 hereinafter, a T1+T5 with a separation of $5 \mathrm{AU}$; Burgasser et al. 2006b, or $\epsilon$ Indi B ab, a T1+T6 separated by 2.65 AU; McCaughrean et al. 2004).

\subsubsection{Spectral Type and Spectral Characteristics}

Figure 6(a) shows the NIR spectrum and $W 1$ and $W 2$ photometry of GU Psc b compared with other objects. The upper panel of the figure shows the spectral standards T2, T3, and T4. The GU Psc b spectrum was smoothed over eight points to a final resolution of $\lambda / \Delta \lambda \sim 400$ to ease comparison. The spectra are from the $\mathrm{L}$ and $\mathrm{T}$ dwarf data archive. $^{12}$ The T2 and T4 (J125453.90-012247.4, hereafter $\mathrm{J} 1254$, and $\mathrm{J} 225418.92+312349.8$ ) are the standards identified in Burgasser et al. (2006a). J120602.51+281328.7 was used as the T3 standard instead of J120956.13-100400.8, which was found to be a binary by Liu et al. (2010). All spectra are normalized to their value at the peak of the $J$ band and are offset vertically for clarity.

The global comparison of the SEDs suggests that GU Psc b is of a spectral type between T2 and T4. The absorption of $\mathrm{CH}_{4}$ in $H$ band is intermediate between that of a T2 and a T4, close to a T3, while the blue side of the $H$ band is better reproduced by the T4 standard. The $Y$ - and $J$-band flux are also closer to the T4, even though in both cases the side of each peak is slightly underluminous. The $K$ band is not similar to any of the standards and is clearly brighter than the average T3 flux. That is probably explained by the collision-induced absorption (CIA) by $\mathrm{H}_{2}$ that affects this region of the spectrum; CIA is expected to be reduced for objects with a lower gravity and/or greater metallicity (Saumon et al. 2012). The $W 1$ flux is lower for GU Psc $\mathrm{b}$ than for any of the standards, and the $W 2$ flux is stronger than the T4, closer to the $\mathrm{T} 2$.

We used the GU Psc b spectrum to compute the spectral indices defined in Burgasser et al. (2006a) and establish the spectral type. The position of the wavelength ranges used and the values derived for the indices are listed in Table 4, along with the associated spectral types for the five indices for which

\footnotetext{
$\overline{12 \text { http://staff.gemini.edu/ sleggett/LTdata.html }}$
} 
Table 4

Spectral Indices for GU Psc b

\begin{tabular}{lcccc}
\hline \hline Index & $\begin{array}{c}\text { Numerator Range } \\
(\mu \mathrm{m})\end{array}$ & $\begin{array}{c}\text { Denominator Range } \\
(\mu \mathrm{m})\end{array}$ & Value & Spectral Type $^{\mathrm{b}}$ \\
\hline $\mathrm{H}_{2} \mathrm{O}-J$ & $1.140-1.165$ & $1.260-1.285$ & $0.30 \pm 0.01$ & $\mathrm{~T} 5$ \\
$\mathrm{CH}_{4}-J$ & $1.315-1.340$ & $1.260-1.285$ & $0.491 \pm 0.009$ & $\mathrm{~T} 4.5$ \\
$W_{J}{ }^{\mathrm{c}}$ & $1.180-1.230$ & $1.260-1.285$ & $0.572 \pm 0.009$ & \\
$\mathrm{H}_{2} \mathrm{O}-H$ & $1.480-1.520$ & $1.560-1.600$ & $0.400 \pm 0.008$ & $\mathrm{~T} 4$ \\
$\mathrm{CH}_{4}-H$ & $1.635-1.675$ & $1.560-1.600$ & $0.73 \pm 0.01$ & $\mathrm{~T} 3$ \\
$\mathrm{H}_{2} \mathrm{O}-K$ & $1.975-1.995$ & $2.08-2.100$ & $0.46 \pm 0.03$ & \\
$\mathrm{CH}_{4}-K$ & $2.215-2.255$ & $2.08-2.120$ & $0.404 \pm 0.009$ & $\mathrm{~T} 3.5$ \\
$K / J$ & $2.060-2.100$ & $1.250-1.290$ & $0.317 \pm 0.006$ & \\
\hline
\end{tabular}

Notes.

${ }^{a}$ The indices are defined as $\int_{a}^{b} f(\lambda) d \lambda / \int_{c}^{d} f(\lambda) d \lambda$, the numerator being the integrated flux in a region inside an absorption feature, the denominator being the integrated flux in an adjacent pseudo continuum.

${ }^{\mathrm{b}}$ The associated spectral type according to Burgasser et al. (2006a).

${ }^{\mathrm{c}}$ For this index, the denominator is multiplied by 2 to compensate for the larger range of the numerator.

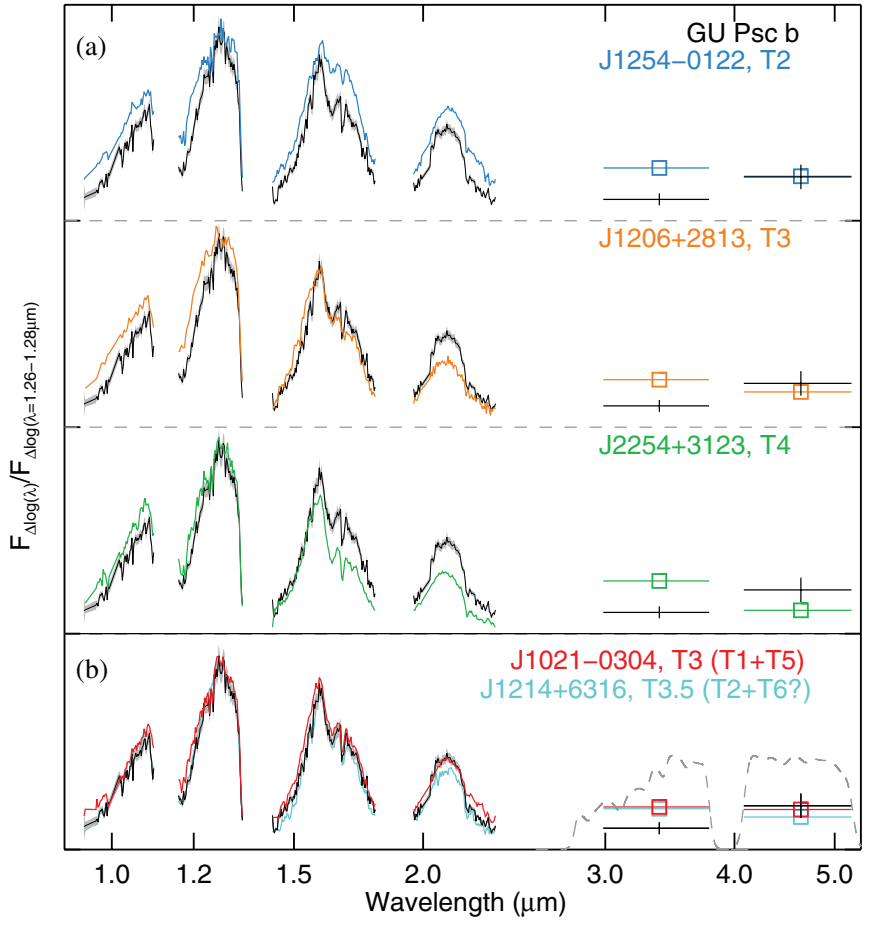

Figure 6. (a) GNIRS NIR spectrum and WISE magnitudes for GU Psc b and the T2, T3, and T4 spectral standards. All SEDs are normalized at the $J$-band peak, between 1.26 and $1.28 \mu \mathrm{m}$. The GU Psc b spectrum has been mediansmoothed to a resolution $\lambda / \Delta \lambda \sim 400$. (b) The GU Psc b SED, now compared to J121440.95+631643.4, a T3.5 that is likely a binary T2+T6, according to the template fitting analysis of Geißler et al. (2011), and J102109.69-030420.1 AB (J1021), the former T3 standard that was confirmed to be a binary T1+T5 by Burgasser et al. (2006b). The GU Psc SED is much closer to that of these objects than to the SED of the standards.

(A color version of this figure is available in the online journal.)

a quantitative scale exists. The $K / J$ index defined by Burgasser et al. (2006a) measures the flux ratio between the $K$ and the $J$ band to evaluate the strength of the CIA- $\mathrm{H}_{2}$ feature that is known to be sensitive to surface gravity. It has no quantitative scale, but the $K / J$ of GU Psc b is stronger than for a typical $\mathrm{T} 3.5$, closer to that of a T2.5 (see $K / J$ versus $\mathrm{CH}_{4}-H$ diagram shown on Figure 10 of Burgasser et al. 2006a). The $W_{J}$ index quantifies the width of the $J$-band peak. The value of GU Psc $\mathrm{b}$ is similar to that we computed for the T4-T5 objects present in the $\mathrm{L}$ and $\mathrm{T}$ dwarf data archive. The resulting spectral types vary between $\mathrm{T} 3$ and $\mathrm{T} 5$, with an average spectral type of $\sim \mathrm{T} 4$.

Altogether, the comparison of the spectrum and the indices suggest that GU Psc b is a T3.5 \pm 1 spectral type, with indication of low gravity, and/or of high metallicity.

Even though GU Psc b is not a resolved binary object according to Keck NIRC2 observations (see Section 3.2.2), the values of its indices, within uncertainties, satisfy two to three of the six spectral index selection criteria developed by Burgasser et al. (2010a) to identify binary systems. GU Psc b would thus qualify as a candidate binary in that scheme (weak candidates satisfy two criteria, and strong ones satisfy three criteria or more). Indeed, as shown on Figure 6(b), the GU Psc $\mathrm{b}$ spectrum is very similar to that of $\mathrm{J} 1021$ (also shown on Figure 6(b)), which was the initial standard for the T3 spectral type (Burgasser et al. 2002), until it was confirmed through Hubble Space Telescope NICMOS imaging to be a $0^{\prime \prime} 172 \pm 0 . \prime 005(5.0 \pm 0.7)$ AU binary composed of a T1+T5 (Burgasser et al. 2006b). The GU Psc b spectrum is also very similar to J121440.95+631643.4 (J1214 hereinafter), a T3.5 discovered by Chiu et al. (2006). Geißler et al. (2011) identified $\mathrm{J} 1214$ as a candidate binary because they obtained a better fit with a composite spectrum made of T2 and T6 templates than for a T3 template. Although GU Psc b remains significantly redder in $J-K_{\mathrm{s}}$, the $Y$ and $J$ bands are closely matched, and the fit is much better in $H$ band. In both cases, the only notable difference lies in the $K_{\mathrm{s}}$-band spectrum. If GU Psc b is truly a single object, this suggests that it is slightly peculiar when compared to standards, perhaps due to the lower gravity expected for a relatively young object, or to some variation in metallicity or cloud properties.

\subsubsection{Direct Comparison with Atmosphere Models}

To further constrain the physical properties of GU Psc b, we compared its NIR spectrum and its $z, W 1$, and $W 2$ photometry to the synthetic SEDs of two different sets of brown dwarf atmosphere models: the BT-Settl CIFIST model presented in Allard et al. (2013) ${ }^{13}$ and the model presented in Morley et al. (2012), which include low-temperature condensates (primarily sulfides). To determine quantitatively the best fit to this mix of photometric and spectroscopic data points, we used a method 13 This grid, which uses the Caffau et al. (2011) solar abundance, is available
online at http://phoenix.ens-lyon.fr/Grids/BT-Settl/CIFIST2011/. 
similar to that presented in Cushing et al. (2008) in order to compute a goodness-of-fit $G_{k}$ for each model $k$ that is minimized for the best fitting models:

$$
G_{k}=\sum_{i=1}^{n} W_{i}\left(\frac{F_{\mathrm{obs}, i}-C_{k} F_{k, i}}{\sigma_{\mathrm{obs}, i}}\right)^{2} .
$$

In this equation, $F_{\mathrm{obs}, i}$ is the flux observed in a given spectral range $i$. The associated uncertainty, $\sigma_{\mathrm{obs}, i}$, is dominated by the $\sim 5 \%$ photometric zero-point uncertainty. The total number of spectral ranges is $n$ : one per photometric filter or spectral point (after smoothing). $F_{k, i}$ is the flux of the synthetic model over the same wavelength domain. The synthetic model spectra are convolved at the resolution of the spectrum and the synthetic photometric magnitudes are obtained using instrumental filter profiles. ${ }^{14}$ Rather than using $W_{i}=\Delta \lambda=\lambda_{2}-\lambda_{1}$ for the weight (as done in Cushing et al. 2008), we chose to use $W_{i}=\Delta \log \lambda=$ $\log \left(\lambda_{2} / \lambda_{1}\right)$ to purposefully not be biased by the arbitrary choice of working in wavelength space rather than frequency space. The scale $C_{k}$ is equal to the dilution factor $R^{2} / d^{2}$ for a source of radius $R$ at a distance $d$. For each model, we constrained $C_{k}$ using the central distance inferred from Bayesian statistical analysis for GU Psc in ABDMG, $d_{s}=48 \mathrm{pc}$, and the radius prescribed at the given $T_{\text {eff }}$ and $\log g$ by evolution models (Saumon \& Marley 2008 for the low-temperature cloud model and Baraffe et al. $2003^{15}$ for BT-Settl).

For the low-temperature cloud model described in Morley et al. (2012), we used a grid with temperatures between $700 \mathrm{~K}$ and $1300 \mathrm{~K}\left(\Delta T_{\text {eff }}=100 \mathrm{~K}\right)$ and $\log g$ between 4.0 and 5.5 $(\Delta \log g=0.5)$, at solar metallicity. We also tried different values for the sedimentation efficiency $f_{\text {sed }}(1-5)$ and for $K_{z z}$, which quantifies departure from chemical equilibrium ( 0 and $10^{4} \mathrm{~cm}^{2} \mathrm{~s}^{-1}$ ). For the BT-Settl model, we used the CIFIST grid presented in Allard et al. (2013), computed with temperatures between $700 \mathrm{~K}$ and $1400 \mathrm{~K}\left(\Delta T_{\text {eff }}=50 \mathrm{~K}\right)$ and $\log g$ between 3.5 and $5.5(\Delta \log g=0.5)$, also at solar metallicity.

Figure 7 shows the goodness-of-fit map for both sets of models in the temperature/log $g$ parameter space. The physical parameters that lead to the best fit between the observed spectrum and the models are the same for both sets, $T_{\text {eff }} \sim$ 1000-1100 $\mathrm{K}$ and $\log g \sim 4.5-5.0$. The observed SED of the object constrains the bolometric luminosity, there is thus a correlation between temperature and gravity for the best fits: they are achieved either at a low temperature $\left(T_{\text {eff }}=\right.$ $1000-1050 \mathrm{~K})$ and low surface gravity $(\log g=4.5)$ or at higher temperature $\left(T_{\text {eff }}=1100 \mathrm{~K}\right)$ and greater gravity $(\log g=5.0)$. In the case of BT-Settl, even higher temperature $\left(T_{\text {eff }}=1200 \mathrm{~K}\right)$ and surface gravity $(\log g=5.5)$ still give a good fit, although these physical parameters are not consistent with the age of ABDMG (see Section 3.2.5). Figure 8 shows the GU Psc b SED and the two best synthetic spectra for both sets of models.

We also did the entire fitting process constraining the $C_{k}$ with the extreme values of the distance range, $43 \mathrm{pc}$ and $53 \mathrm{pc}$. The effective temperature and surface gravity we obtained did not vary significantly.

Both synthetic models match the SED of GU Psc b between 0.9 and $5 \mu \mathrm{m}$ remarkably well, especially considering that there

\footnotetext{
14 The GMOS-South $z$ transmission curve available at

http://www.gemini.edu/sciops/instruments/gmos/filters/gmos_s_z_G0328.txt

is convolved with the detector response (http://www.gemini.edu/sciops/

instruments/gmos/imaging/detector-array/gmoss-array). The WISE transmission curves are taken from Wright et al. (2010).

15 Available at http://phoenix.ens-lyon.fr/Grids/BT-Settl/CIFIST2011/ ISOCHRONES/.
}
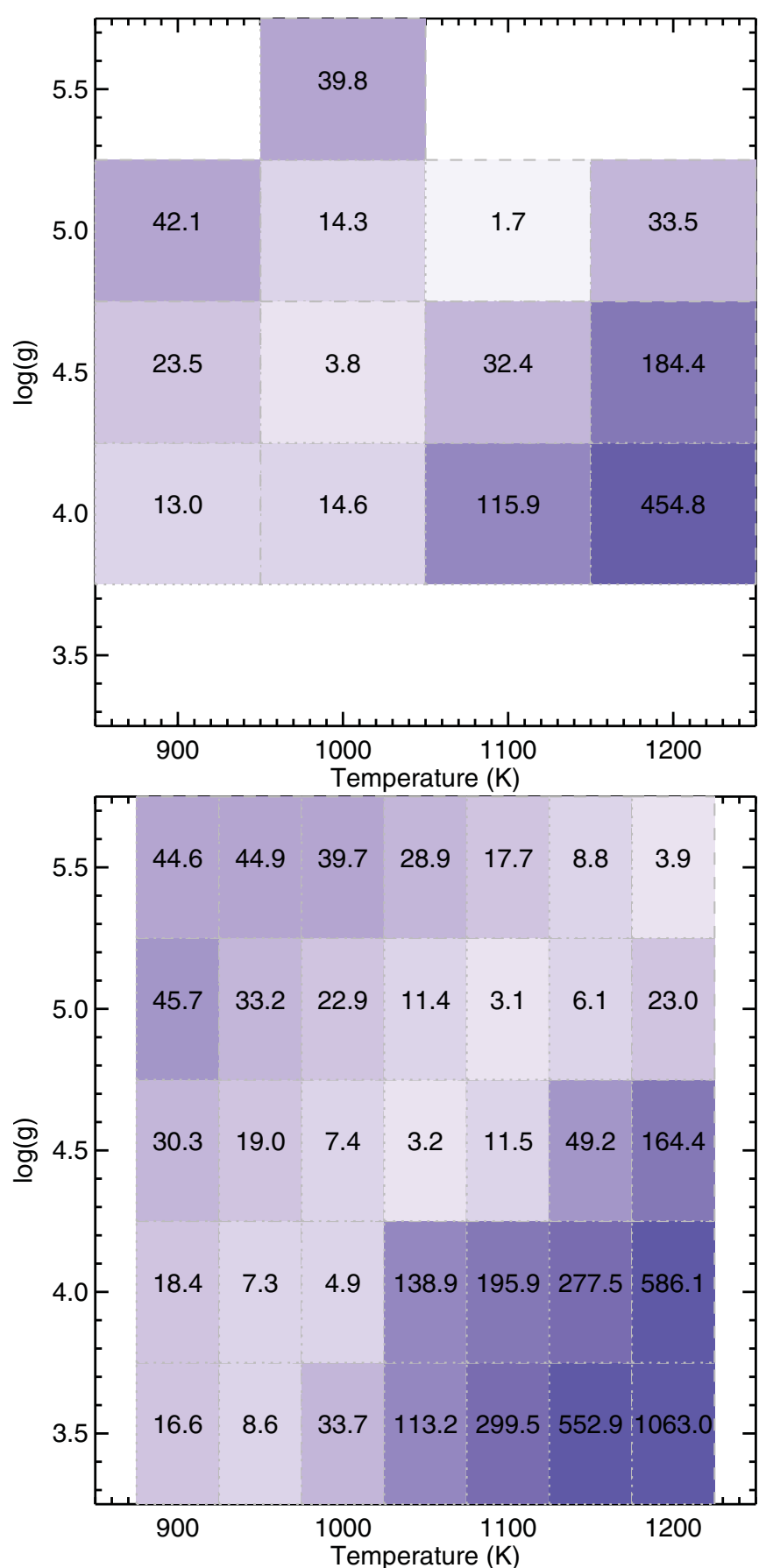

Figure 7. Goodness-of-fit maps for the low-temperature cloud model of Morley et al. (2012) (upper panel) with $f_{\text {sed }}=1$ and $K_{z z}=0 \mathrm{~cm}^{2} \mathrm{~s}^{-1}$ and the BT-Settl model (lower panel), using the same contour levels. The value of the goodnessof-fit $G$ is written for each set of parameters. Both sets of models reach a minimum around $T_{\text {eff }}=1000-1100 \mathrm{~K}$ and $\log g=4.5-5.0$. These results are obtained with the scale $C_{k}$ constrained using the radius from evolutionary models (Saumon \& Marley 2008 for the low-temperature cloud model and Allard et al. 2013 for the BT-Settl) and $d_{s}=48 \mathrm{pc}$ for the distance.

(A color version of this figure is available in the online journal.)

is no adjustment of the absolute flux of the models $\left(C_{k}\right.$ is constrained). Overall, BT-Settl fits the $K$ band (especially its red side) and the $Y$ band around $1 \mu \mathrm{m}$ slightly better than the low-temperature cloud model, but the later one better reproduces the $J-H$ color. The methane band at $1.6 \mu \mathrm{m}$ is typically poorly matched by the models which tend to overestimate the flux in this wavelength range (see e.g., HN Peg b SED on Figure 4 of 

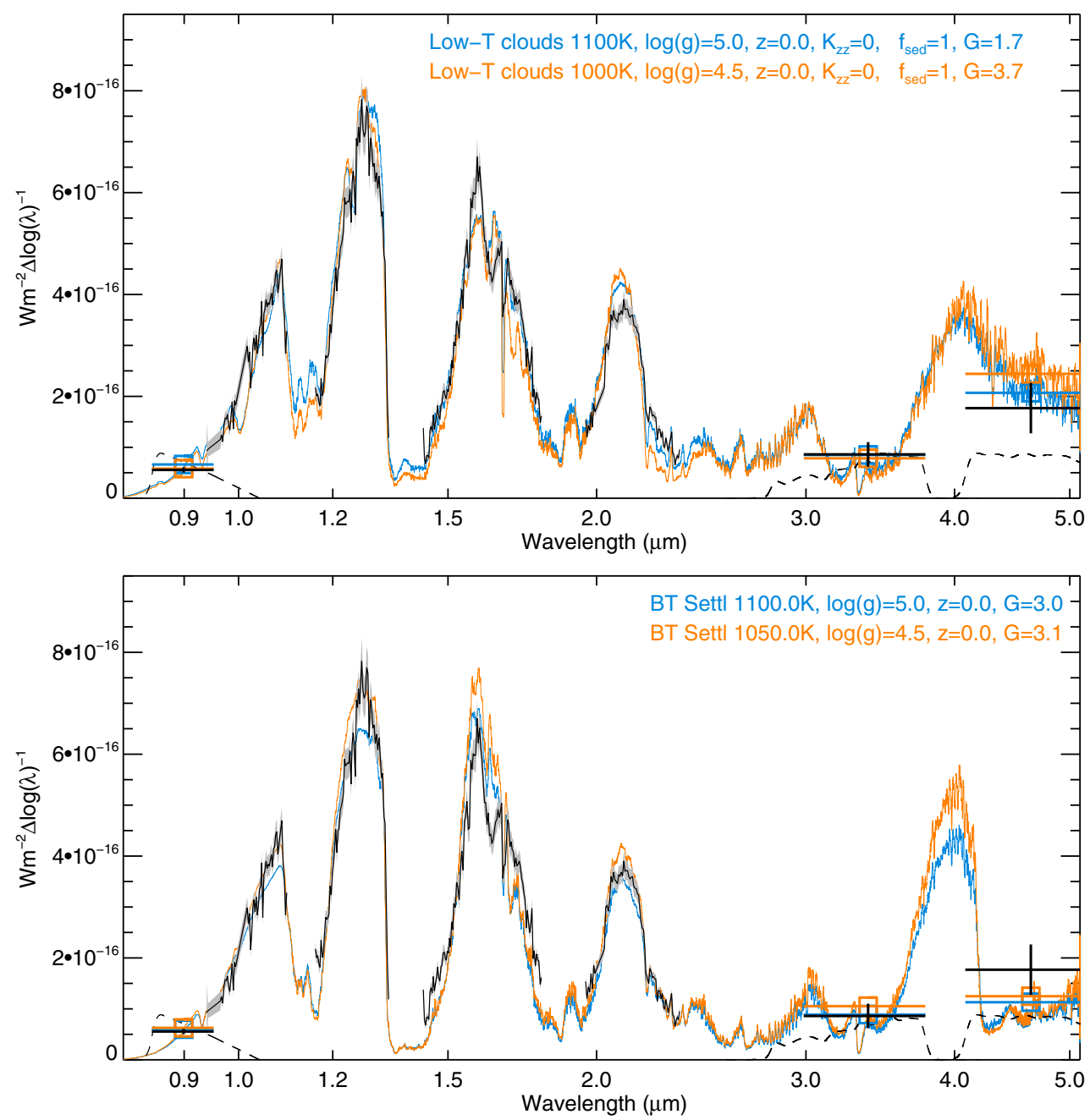

Figure 8. GU Psc b GNIRS NIR spectrum and $z, W 1$, and $W 2$ photometry points along with the best-fit model spectra for the low-temperature cloud model of Morley et al. (2012) (upper panel) and the BT-Settl model (lower panel). For each model, the parameters (effective temperature $T_{\text {eff }}$, surface gravity log $g$, metallicity $z$, the Eddy coefficient $K_{z z}$, and the sedimentation parameters $f_{\text {sed }}$ ) are given. The goodness-of-fit $G$, which allows us to quantify the quality of the fit (the smaller $G$ is, the better the fit; see Section 3.2.4) is also shown. The model flux scale is absolute, using the radius from evolutionary models (Saumon \& Marley 2008 for the low-temperature cloud model, and Allard et al. 2013 for the BT-Settl) for every set of parameters, and the statistical distance of the primary in ABDMG, $d_{s}=48$ pc.

(A color version of this figure is available in the online journal.)

Leggett et al. 2008). Here, we observe an opposite trend: the flux redward of $1.7 \mu \mathrm{m}$ is too low in both sets of models compared to GU Psc b. This excess flux, which is also seen in the comparison of GU Psc b to the T3 and T4 templates on Figure 6(a), could be indicative of a slight departure from solar metallicity or an inhomogeneous surface, or an indication that GU Psc b is a very tight, unresolved, binary object.

The good fit we obtain with Morley et al. (2012) model is somewhat surprising, considering our object is hotter than the targeted objects for this model. This model came as an attempt to explain why late $\mathrm{T}$ dwarfs $\left(T_{\text {eff }} \lesssim 900 \mathrm{~K}\right.$, primarily) were not perfectly fitted with models without iron and silicate clouds. Using Ackerman \& Marley (2001) cloud model, they studied the influence of other condensates (sulfides mainly, e.g., $\mathrm{Na}_{2} \mathrm{~S}, \mathrm{MnS}$, and $\mathrm{ZnS}$, but also $\mathrm{KCl}$ and $\mathrm{Cr}$ ) that were previously ignored and realized they are important at low temperatures (between 400 and $1300 \mathrm{~K}$ ). Since the targeted objects are late T dwarfs, Morley et al. (2012) models do not include the iron and silicate clouds that are important for L dwarfs since they are thought to be rapidly clearing at the $\mathrm{L} / \mathrm{T}$ transition. In models including such condensates (but not the ones included in the Morley et al. 2012 model), early T such as GU Psc b are expected to be best described by thin clouds of larger particles (corresponding to a high $f_{\text {sed }}$ parameter in the Ackerman \& Marley 2001 model). This is the case, for example, for HN Peg b (T2.5), for which the best fit is obtained with $f_{\text {sed }}=3.5$ (Leggett et al. 2008) or for J1254 (T2) or 2M J05591914-1404488 (T4.5), which were best fit with $f_{\text {sed }}=3$ and 4 , respectively, in Cushing et al. (2008). Alternatively, our best fits with Morley et al. (2012) model are obtained using $f_{\text {sed }}=1$, thus very thick clouds of the less abundant sulfide condensates provide the moderate dust opacity evident in our T3.5 object. Figure 9 compares one of our best fits using $f_{\text {sed }}=1$ (in blue) and a much poorer fit with the same temperature/ $\log g$ but with thinner sulfide clouds $\left(f_{\text {sed }}=3\right.$, in green $)$.

The second parameter is the Eddy diffusion coefficient, $K_{z z}$ that characterizes the vertical transport in the atmosphere. Vigorous vertical transport can bring molecular species from deeper, hotter layers of the atmosphere to the upper, cooler layers on a time scale faster than that of some chemical reactions, driving the molecular abundances away from their local equilibrium values. In particular, this results in increased $\mathrm{CO}$ and $\mathrm{CO}_{2}$ abundances, and reduced $\mathrm{CH}_{4}, \mathrm{H}_{2} \mathrm{O}$, and $\mathrm{NH}_{3}$ abundances in the upper atmosphere (Lodders 2002; Saumon 


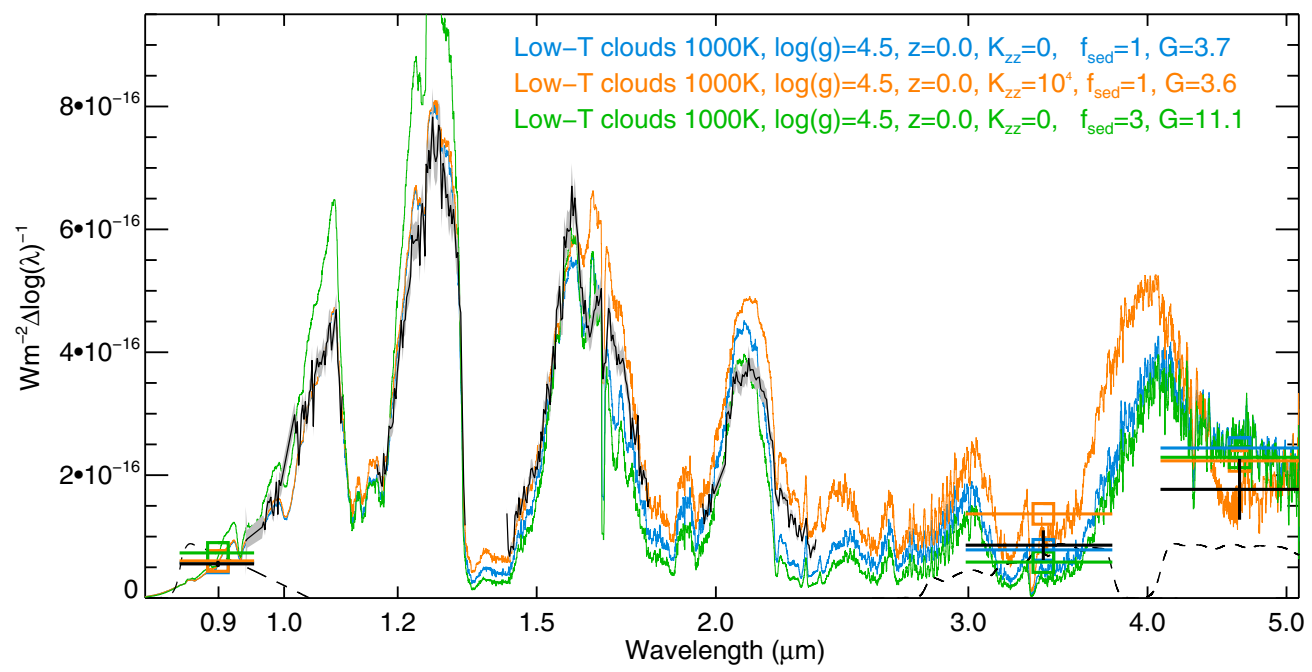

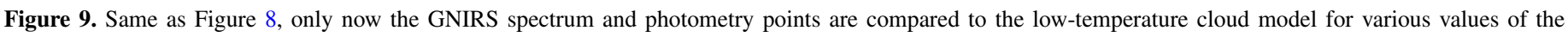
parameters $f_{\text {sed }}$ and $K_{z z}$.

(A color version of this figure is available in the online journal.)

et al. 2006; Burningham et al. 2011). Figure 9 shows, for $f_{\text {sed }}=$ 1 , the two available values we tested for the Eddy diffusion coefficient: $K_{z z}=0 \mathrm{~cm}^{2} \mathrm{~s}^{-1}$ (chemical equilibrium, in blue) and $K_{z z}=10^{4} \mathrm{~cm}^{2} \mathrm{~s}^{-1}$ (in orange). It shows that $K_{z z}$ has little impact on the $Y$ and $J$ bands, but that an increase in $K_{z z}$ increases the flux in $H, K$ and in the mid-infrared. The best fit at $K_{z z}=0 \mathrm{~cm}^{2} \mathrm{~s}^{-1}$ is obtained at $T_{\text {eff }}=1100 \mathrm{~K}$ and $\log g=5.0(G=1.7$, Figure 8 , in blue) while at $K_{z z}=10^{4} \mathrm{~cm}^{2} \mathrm{~s}^{-1}$, the best fit is obtained at a slightly lower temperature and $\log g: T_{\text {eff }}=1000 \mathrm{~K}$ and $\log g=$ $4.5(G=3.6$, Figure 9 , in orange). The depth of the $4.6 \mu \mathrm{m}$ absorption band in $W 2$ is reproduced a bit better with a higher values of $K_{z z}$. We must caution that we did not try a higher $K_{z z}$ for values of $f_{\text {sed }}$ different than 1 .

The BT-Settl model treats condensation and sedimentation of all dust species as well as gas phase advection by relating to a single atmospheric velocity field derived from radiation hydrodynamical models. There are thus no adjustable cloud parameters in this model. The cloud sedimentation and Eddy diffusion are instead determined by direct comparison of the relevant timescales (for condensation, sedimentation, chemical reactions) to the mixing timescale derived from this velocity field. The cloud opacity is composed of a number of condensates, which are settling to various degrees. Although the current version of the model does not yet include the opacity contribution of all low-temperature condensates that are included in Morley et al. (2012) (most notably $\mathrm{Na}_{2} \mathrm{~S}$ ), the BT-Settl model reproduces the observed spectrum of GU Psc b similarly well. This is because even if these low-temperature clouds start to form at high altitude for L/T transition objects like GU Psc b, they do not become optically thick in this effective temperature range. The continuum of the flux peaks is still shaped by the silicate clouds, even if they have receded relatively deep into the photosphere.

The excess absorption at 4-5 $\mu \mathrm{m}$ apparent for this model could be indicative of an overestimation of the diffusion efficiency, resulting in too much $\mathrm{CO}$ and $\mathrm{CO}_{2}$ being mixed into the photosphere. The mixing derived at the transition from $\mathrm{CO}$ to $\mathrm{CH}_{4}$-dominated atmosphere regions yields a diffusion coefficient of $\sim 10^{5}-10^{6} \mathrm{~cm}^{2} \mathrm{~s}^{-1}$. It thus produces more $\mathrm{CO}$ and $\mathrm{CO}_{2}$ absorption at 4-5 $\mu \mathrm{m}$ than the $K_{z z}=0$ or the $K_{z z}=10^{4}$ models of Morley et al. (2012). Alternatively, the excess flux in the $\mathrm{CH}_{4}$ absorption band in $W 1$ might also reveal the incompleteness of the currently used $\mathrm{CH}_{4}$ opacities, which cover only a fraction of the lines relevant at temperatures above $1000 \mathrm{~K}$ (Yurchenko \& Tennyson 2014).

We investigated the effect of leaving the scale $C_{k}$ free in the fitting. For both synthetic models, for a given set of parameters, we then obtain a similar or slightly better fit than when imposing the scale. Nonetheless, the best fit occurs for the same physical parameters than with a constrained $C_{k}$. This strengthens our confidence both in the radii predicted by evolutionary models and in the distance inferred by the Bayesian statistical analysis.

\subsubsection{Physical Properties from Evolutionary Models}

Evolutionary models can be used to constrain the physical parameters (radii, surface gravity, bolometric luminosity, and mass) of GU Psc b. In the previous section, we showed that the best fit were obtained with $T_{\text {eff }} / \log g$ couples of $1000-1050 \mathrm{~K} /$ 4.5 or $1100 \mathrm{~K} / 5.0$. Evolutionary models suggest that the lowest temperature and $\log g$ are the most likely; the age is then consistent with the age of ABDMG, $100 \pm 30$ Myr. The highest temperature and $\log g$ would imply ages older than $\sim 300$ Myr. The age deduced from evolutionary models for $1200 \mathrm{~K} / 5.5$, which also led a good fit for BT-Settl, are greater than $1 \mathrm{Gyr}$ and are clearly excluded for ABDMG age.

Thus, using ABDMG age $(100 \pm 30 \mathrm{Myr})$ and the range of plausible temperatures determined with atmosphere models $\left(T_{\text {eff }}=1000-1100 \mathrm{~K}\right)$ in evolutionary models, we obtain the ranges of values for the physical properties of GU Psc b presented in Table 5. We used the two different models presented in Saumon \& Marley (2008): one with $f_{\text {sed }}=2$, which is a good approximation for all cloudy models, and one without clouds. We present both results as limiting cases in Table 5, but since the atmosphere model fitting suggests a better match with clouds than without, the cloudy version is probably the most appropriate for GU Psc b. We also used the evolutionary model of Baraffe et al. (2003) and obtained similar results. In all cases, the values obtained for the bolometric luminosity are between $\log \left(L / L_{\odot}\right)=-4.9$ and -4.6 . The surface gravity inferred $(\log g=4.2-4.4)$ is consistent, albeit slightly lower, with the values derived from atmosphere model fitting $(\log g=4.5-5)$. All models suggest a mass between 9 and 
Table 5

Evolutionary Model Derived Physical Properties of GU Psc $b^{\mathrm{a}}$

\begin{tabular}{lcccc}
\hline \hline $\begin{array}{l}\text { Property } \\
\text { Model }\end{array}$ & $\begin{array}{c}R \\
\left(R_{\text {Jup }}\right)\end{array}$ & $\log g$ & $\log \left(L / L_{\odot}\right)$ & $\begin{array}{c}\text { Mass } \\
\left(M_{\text {Jup }}\right)\end{array}$ \\
\hline S08 cloudy $^{\mathrm{b}}$ & $1.33-1.38$ & $4.18-4.23$ & $-4.80-4.60$ & $10.8-12.0$ \\
S08 no cloud $^{\mathrm{b}}$ & $1.23-1.27$ & $4.23-4.31$ & $-4.87-4.67$ & $10.4-12.1$ \\
B03 $^{\mathrm{c}}$ & $1.15-1.21$ & $4.22-4.36$ & $-4.91-4.70$ & $9.6-13.0$ \\
\hline
\end{tabular}

Notes.

a Assuming an age of $100 \pm 30 \mathrm{Myr}$ and a $T_{\text {eff }}=1000-1100 \mathrm{~K}$.

${ }^{\mathrm{b}}$ Using Saumon \& Marley (2008) evolutionary models. The cloudy version is with $f_{\text {sed }}=2$, and is also appropriate for $f_{\text {sed }}=1$ (see the text).

${ }^{c}$ Using Baraffe et al. (2003) evolutionary models.

$13 M_{\text {Jup }}$. We thus find that GU Psc b is probably below the lower threshold of deuterium burning for its $T_{\text {eff }}$ and age, unlike 2M0122 and several other brown dwarfs discussed in Bowler et al. (2013) that are possible deuterium burners.

\section{ANALYSIS AND DISCUSSION}

\subsection{Stability of the System}

This system has a very wide projected separation $(r=$ $2000 \pm 200 \mathrm{AU}$ ) that is not seen in any other planet-star system (excluding companions to white dwarf or other evolved systems such as WD 0806-661, Luhman et al. 2011; LSPM1459+0857, Day-Jones et al. 2010 or sdM1.5+WD Wolf 1130, Mace et al. 2013 for which stellar mass loss most likely had an impact on the separation). The mass ratio of GU Psc system $(q \sim 0.03)$ is higher than that of typical exoplanetary systems. The value is particularly high for an M dwarf host, these stars seem to be an uncommon host for Jupiter-mass companions, even at close separations (Bonfils et al. 2013). However, it is significantly lower than the mass ratio of several directly imaged systems, such as the $30 M_{\text {Jup }}$ brown dwarf around the M1 star CD-35 2722 ( $q \sim 0.07$; Wahhaj et al. 2011) or the $4 M_{\text {Jup }}$ around the M8 brown dwarf 2M 1207 ( $q \sim 0.15$; Chauvin et al. 2004).

The very large separation coupled to the very low mass ratio led to a very small binding energy. With a primary mass of $M_{\star}=0.30-0.35 M_{\odot}$, using $M_{c}=9-13 M_{\text {Jup }}$ for the mass of the companion and $r=2000 \mathrm{AU}$ as the instantaneous projected separation, the binding energy of the system is (assuming a circular orbit)

$$
E_{\text {bind }} \sim-\frac{G M_{\star} M_{c}}{1.27 r}=-(0.2 \pm 0.1) \times 10^{41} \mathrm{erg},
$$

where 1.27 is the average projection factor between $r$ and the semimajor axis, assuming a random viewing angle, see, e.g., Brandeker et al. (2006). Although this binding energy is very small, it is the same order of magnitude as that of other presumably gravitationally bound systems that include a planetary-mass companion, such as Ross $458(\mathrm{AB}) \mathrm{c}$ system $\left(E_{\text {bind }} \sim-1 \times 10^{41} \mathrm{erg}\right.$, using $r \sim 1168 \mathrm{AU}, M_{\star} \sim 0.61 M_{\odot}$, and $M_{c} \sim 14 M_{\text {Jup }}$; Goldman et al. 2010) or the 2M1207 A brown dwarf (BD) and its companion $\left(E_{\text {bind }} \sim-0.2-0.6 \times 10^{41} \mathrm{erg}\right.$, using $r \sim 52 \mathrm{AU}, M_{\mathrm{BD}} \sim 25 M_{\mathrm{Jup}}$, and $M_{c}=3-10 M_{\mathrm{Jup}}$; Chauvin et al. 2004; Ducourant et al. 2008). It is also similar to the binding energy of older, more massive systems such as the M3 star G204-39 and T6.5 brown dwarf 2MASS J1758+4633 ( $E_{\text {bind }} \sim-0.4-0.7 \times 10^{41} \mathrm{erg}$, using the masses and separation given in Faherty et al. 2010) and greater than many of the very low mass star systems presented in Dhital et al. (2010). Thus, it is not unreasonable for GU Psc b to be gravitationally bound to its primary despite its very wide separation.

Indeed, one can estimate the average survival time of the system considering the encounters with stars and giant molecular clouds, which are the most important sources of disruption. The chance of disruption depends mainly on the binding energy, so the results shown in Figure 2(a) of Weinberg et al. (1987) for a $1 M_{\odot}$ system can be scaled down for GU Psc system. With a projected separation of $r \sim 2000 \mathrm{AU}\left(\sim 9.7 \times 10^{-3} \mathrm{pc}\right)$ and a total mass of $\sim 0.35 M_{\odot}$, our system lies between the $a_{0}=0.02 \mathrm{pc}$ and $a_{0}=0.04 \mathrm{pc}$ curves, which implies a half-life for the system of $\sim 5-6$ Gyr, which is much greater than its estimated age.

\subsection{Formation Mechanisms}

With such a great distance from its host star and a relatively high mass ratio, it is unlikely that GU Psc b was formed alone in situ in a protoplanetary disk, through one of the canonical formation mechanisms for exoplanets (core accretion; Pollack et al. 1996; Inaba et al. 2003 or disk instability; Bate et al. 2002; Boss 2006; Stamatellos et al. 2007; Rafikov 2009). It would require too large a protoplanetary disk, with an unrealistically large density at this separation.

A plausible scenario is that GU Psc b formed in a disk but migrated outward due to dynamic interactions with an unseen, more massive companion to GU Psc A (Reipurth \& Clarke 2001). Similar low-mass, wide companions have been found around binary star (e.g., Ross 458(AB) c, at >1100 AU, SR 12 $\mathrm{AB} \mathrm{c}$, at $>1000 \mathrm{AU})$. This hypothesis would imply that GU Psc A has a more massive, closer-in companion that was not seen in our observations (see Figure 4). More high-contrast imaging observations would be useful to better constrain the binary nature of GU PSc A. It would also be desirable to assess theoretically whether the hypothetical triple systems allowed in Figure 4 represent realistic dynamical stable solutions, given the age of the system.

Another possibility is that GU Psc A and b were both formed in the disk of a more massive star and have been ejected as a system (Bate et al. 2003; Stamatellos \& Whitworth 2009).

Both components of the system could also have been formed by turbulent core fragmentation, as a weakly bound binary system, similar to a binary star system. It would imply that cores can fragment into objects as light as the companion (which is plausible, given the opacity limit for fragmentation is a few Jupiter masses; Bate 2009) or that the system was ejected from the accretion reservoir following a dynamical interaction (Reipurth \& Clarke 2001; Bate et al. 2002; Bate \& Bonnell 2005).

GU Psc b could also have been a free-floating planet, formed by turbulent core fragmentation or ejection from a protoplanetary system, later captured by GU Psc A (Perets $\&$ Kouwenhoven 2012). In this case, we would observe less correlations between the physical properties (e.g., metallicities or spin-orbit relation) of the primary and secondary. More insight on the possible formation mechanism could thus possibly be obtained by determining the relative metallicity of both components through high-resolution spectroscopy.

\subsection{Interest of the System}

Figure 10 compares the masses, ages, and temperatures of GU Psc b and other low-mass companions. The temperature derived from atmosphere models for GU Psc b and the age of 


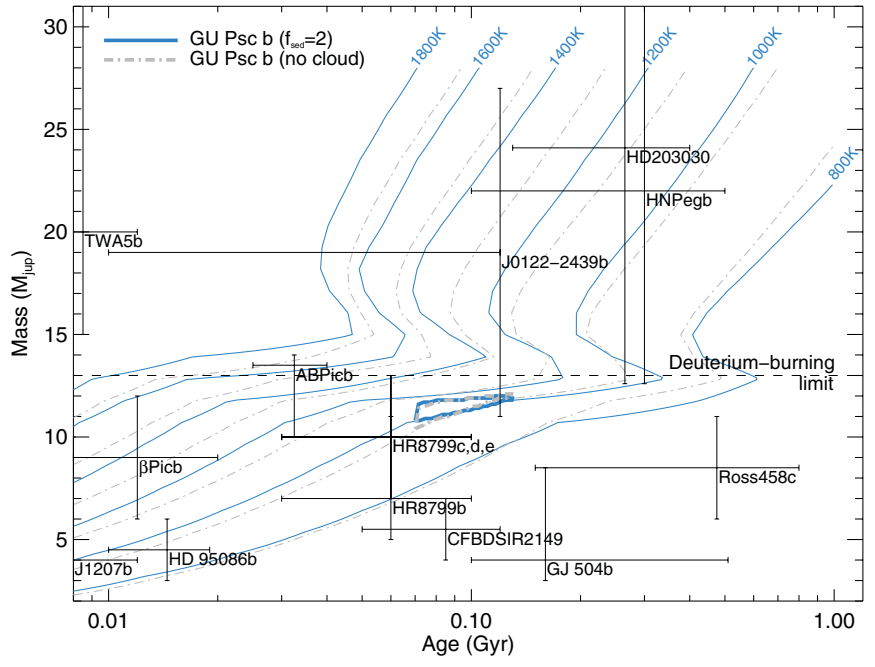

Figure 10. Masses and ages of various low-mass companions. Evolutionary models of Saumon \& Marley (2008) with and without clouds are represented by the solid blue line and the dot-dashed gray line, respectively. Given the age of the ABDMG and the effective temperature range found using atmosphere models $\left(T_{\text {eff }}=1000-1100 \mathrm{~K}\right)$, the mass of GU Psc b is below $13 M_{\text {Jup }}$, irrespective of the model used (10-12 M Jup for Saumon \& Marley 2008 model). Ages and masses for other sources are taken from Bonnefoy et al. (2010), Bowler et al. (2013), Burningham et al. (2011), Chauvin et al. (2004), Ducourant et al. (2008), Kuzuhara et al. (2013), Lagrange et al. (2010), Liu et al. (2011), Lowrance et al. (1999), Luhman et al. (2007), Marois et al. (2008, 2010), Metchev \& Hillenbrand (2006), Neuhäuser \& Schmidt (2012), Rameau et al. (2013), and Delorme et al. (2012).

(A color version of this figure is available in the online journal.)

ABDMG yield a mass below the deuterium-burning mass limit in evolutive models (the Saumon \& Marley 2008 model is shown on Figure 10).

Figure 10 also shows that GU Psc b is intermediate in age between the few planetary-mass objects uncovered in very young associations (e.g., the 8-20 Myr old planet 2M1207 b in TWA or the 12 Myr-old $\beta$ Pictoris b) and the field planetary-mass objects of hundreds Myr and more (e.g., the cool 150-800 Myr Ross $458(\mathrm{AB}) \mathrm{c})$. While the constraints on the age of many companions come from the poorly known age of the system, the ABDMG membership of GU Psc provides much better constraints to the age of the companion, allowing a better validation of the models, still poorly constrained for this mass and age range.

The similarity in age and mass between GU Psc b and the HR 8799 planets is obvious on Figure 10. Indeed, the physical properties of GU Psc b ought to be very similar to the most massive planets that should be discovered by forthcoming planet finder instruments such as the Gemini Planet Imager (Macintosh et al. 2012), SPHERE on the VLT (Beuzit et al. 2008), or the HiCIAO on Subaru (Suzuki et al. 2009), albeit at much closer separations. GU Psc b could serve as a proxy for these planets, that will likely be only characterizable with low-resolution spectroscopy $(R \sim 40)$, due to their proximity to their parent star. GU Psc b, at $\sim 42^{\prime \prime}$ from its primary, will be amenable to detailed follow-up photometric and spectroscopic observations, just like the free-floating planetary-mass object CFBDSIR2149, also a strong candidate member of ABDMG (4-7 $M_{\text {Jup }}$; Delorme et al. 2012).

GU Psc b follows the trend of companions like 2M1207b, 2M 0122B, the planets of HR 8799 and HN Peg B that all show effective temperatures under that of typical field brown dwarfs for a given spectral type (see Bowler et al. 2013, Figure 13). This is likely explained by the lower surface gravity of these objects. GU Psc b provides an important data point to clarify the spectral type versus temperature relationship at intermediate ages.

With its spectral type of T3.5 \pm 1 , a derived temperature between 1000 and $1100 \mathrm{~K}$ and an age of 70-130 Myr, GU Psc $\mathrm{b}$ is a rare example of a young early $\mathrm{T}$ dwarfs straddling the $\mathrm{L} / \mathrm{T}$ transition between the cloudy and clear regime of brown dwarf atmospheres. The $\mathrm{L} / \mathrm{T}$ transition is particularly challenging for atmosphere models because of the complex treatment of clouds required. At this transition, it is expected that the iron/silicate clouds, important source of opacity for L dwarfs (Saumon \& Marley 2008; Stephens et al. 2009), gradually become less important, either because they sink or become patchy, which allows the emergent flux to come from a deeper layer of the atmosphere. As suggested in Morley et al. (2012), other condensate could become important for cooler mid- to late-T dwarfs. As shown in Section 3.2.4, GU Psc b's SED is well reproduced both by the BT-Settl model (Allard et al. 2012, 2013), that includes iron/silicate condensates (and some of the low-temperature condensates), and by the Morley et al. (2012) model that includes sulfides and other low-temperature condensates, using thick clouds $\left(f_{\text {sed }}=1\right)$.

Several early field $\mathrm{T}$ dwarfs are known to show a photometric variability that could, in fact, be explained by a combination of cloudy and clear regions in the atmosphere, or by a partial cloud cover. For GU Psc b, our limited set of three $J$-band images (spanning $\sim 11$ months; see Table 1 ) gives magnitudes that are all consistent with each other within $1 \sigma$. These data set a $3 \sigma$ upper limit on variability of approximately $150 \mathrm{mmag}$. GU Psc $\mathrm{b}$ would be a prime target for further photometric variability studies. Although it is much fainter than other field early $\mathrm{T}$ dwarfs, variability studies of GU Psc b are well within the capability of existing ground- and space-based telescopes.

The binary hypothesis being to a large extent ruled-out for the companion, it would be interesting to extend the analysis with models atmospheres and spectra for partly cloudy atmospheres (Marley et al. 2010) or even composite spectra for atmosphere models with horizontal temperature variations.

\section{SUMMARY AND CONCLUSIONS}

We have presented the discovery of a comoving planetarymass companion to GU Psc, a low-mass M3 star and strong candidate member of the $\sim 100 \mathrm{Myr}$ old ABDMG association. We presented evidences that strongly support a membership in ABDMG. Notably, its kinematics and X-ray emission fit that of the association. The companion is widely separated from the host star at $\sim 42^{\prime \prime}$ or $\sim 2000 \mathrm{AU}$ at the estimated distance of $48 \mathrm{pc}$.

The companion has the spectral signature of a T3.5 \pm 1 spectrum, with relatively strong $K$-band emission, a likely indicator of a low-gravity object. The overall SED resembles closely that of a binary T0+T5 (J1021) and of a candidate binary T2+T6 (J1214) spectra, but it has been shown with Keck LGS AO observations that it is in all likelihood a "single" early T3.5 dwarf. Few such L/T transition dwarfs are known. They constitute particularly interesting candidates for variability studies as they are likely to have partial and variable cloud cover. GU Psc b is a prime target to extend previous photometric variability studies of old early $\mathrm{T}$ dwarfs to younger ages.

Astrometric observations, through the CTIOPI ${ }^{16}$ project, are ongoing to secure the parallax of the system. A precise

\footnotetext{
16 http://www.chara.gsu.edu/ thenry/CTIOPI/
} 
distance will confirm the membership of the primary, hence confirm the age of the system, and allow to better establish the physical parameters of the companion. High-contrast imaging observations of the host star could put better constraints on the mass ratio and separation of an hypothetical closer companion to the star. The mid-infrared spectroscopy of GU Psc b could provide significant constraints to atmosphere models in this region. It will be an easy target for NIRSpec and MIRI on board the James Webb Space Telescope (Gardner et al. 2006). GU Psc $\mathrm{b}$ should become an excellent proxy for relatively massive gas giant planets soon to be discovered by forthcoming high-contrast imaging instruments.

We would like to thank the anonymous referee for constructive comments and suggestions that greatly improved the overall quality of the paper. This work was financially supported by the Natural Sciences and Engineering Research Council (NSERC) of Canada and the Fond de Recherche Québécois-Nature et Technologie (FRQNT; Québec). D.S. is supported by NASA Astrophysics Theory grant NNH11AQ54I. D.H. acknowledges support from the European Research Council under the European Community's Seventh Framework Programme (FP7/2007-2013 Grant Agreement no. 247060). Based on observations obtained at the Gemini Observatory (Gemini-S/ PHOENIX: program GS-2010B-Q-89, Gemini-S/GMOS: program GS-2011B-Q-74, Gemini-S/NICI: programs GS-2011BQ-24 and GS-2012B-Q-54 and Gemini-N/GNIRS: program GN-2012B-Q-58), which is operated by the Association of Universities for Research in Astronomy, Inc., under a cooperative agreement with the NSF on behalf of the Gemini partnership: the National Science Foundation (United States), the National Research Council (Canada), CONICYT (Chile), the Australian Research Council (Australia), Ministerio da Cincia, Tecnologia e Inovacao (Brazil) and Ministerio de Ciencia, Tecnologa e Innovacin Productiva (Argentina). Observations were also collected at CFHT with WIRCam (run IDs: $11 \mathrm{BC} 20$ and 12BC20) and ESPaDOnS (run ID: 12AC23), at the European Southern Observatory Very Large Telescope under program ID: 087.D-0510, 091.D-0641 and on CPAPIR infrared camera, at Observatoire du mont Mégantic, which is funded by the Université de Montréal, Université Laval and the Canada Foundation for Innovation. Some of the data presented herein were obtained at the W. M. Keck Observatory, which is operated as a scientific partnership among the California Institute of Technology, the University of California, and the National Aeronautics and Space Administration. The Observatory was made possible by the generous financial support of the W. M. Keck Foundation. The authors recognize and acknowledge the very significant cultural role and reverence that the summit of Mauna Kea has always had within the indigenous Hawaiian community. We are most fortunate to have the opportunity to conduct observations from this mountain. Finally, we also obtained data from the NASA InfraRed Telescope Facility, with SpeX, under the program number 2013B025. This publication makes use of data products from the Wide-field Infrared Survey Explorer, which is a joint project of the University of California, Los Angeles, and the Jet Propulsion Laboratory/California Institute of Technology, funded by the National Aeronautics and Space Administration, from the Two Micron All Sky Survey, which is a joint project of the University of Massachusetts and the Infrared Processing and Analysis Center, and funded by the National Aeronautics and Space Administration and the National Science Foundation, of the NASA's Astrophysics Data System Bibliographic Ser- vices, SIMBAD database, the VizieR catalog access tool and the SIMBAD database operated at CDS, Strasbourg, France. The BT-Settl model atmospheres have been computed at the Pôle Scientifique de Modélisation Numérique of the ENS de Lyon, and at the Gesellschaft für Wissenschaftliche Datenverarbeitung Göttingen in co-operation with the Institut für Astrophysik Göttingen. This publication has made use of the $\mathrm{L}$ and $\mathrm{T}$ dwarf data archive, http://staff.gemini.edu/ sleggett/LTdata.html.

\section{REFERENCES}

Ackerman, A. S., \& Marley, M. S. 2001, ApJ, 556, 872

Ahn, C. P., Alexandroff, R., Allende Prieto, C., et al. 2012, ApJS, 203, 21

Albert, L., Artigau, É., Delorme, P., et al. 2011, AJ, 141, 203

Allard, F., Homeier, D., \& Freytag, B. 2012, RSPTA, 370, 2765

Allard, F., Homeier, D., Freytag, B., Schaffenberger, W., \& Rajpurohit, A. S. 2013, MSAIS, 24, 128

Artigau, É., Biller, B. A., Wahhaj, Z., et al. 2008, Proc. SPIE, 7014, 70141

Artigau, É., Doyon, R., Vallée, P., Riopel, M., \& Nadeau, D. 2004, Proc. SPIE, 5492, 1479

Artigau, É., Lafrenière, D., Doyon, R., et al. 2011, ApJ, 739, 48

Artigau, É., Lamontagne, R., Doyon, R., \& Malo, L. 2010, Proc. SPIE, 7737,41

Baraffe, I., Chabrier, G., Allard, F., \& Hauschildt, P. H. 1998, A\&A, 337, 403

Baraffe, I., Chabrier, G., Barman, T. S., Allard, F., \& Hauschildt, P. H. 2003, A\&A, 402, 701

Barenfeld, S. A., Bubar, E. J., Mamajek, E. E., \& Young, P. A. 2013, ApJ, 766, 6

Barman, T. S., Macintosh, B., Konopacky, Q. M., \& Marois, C. 2011, ApJ, 733, 65

Barrado y Navascués, D., \& Martín, E. L. 2003, AJ, 126, 2997

Bate, M. R. 2009, MNRAS, 392, 590

Bate, M. R., \& Bonnell, I. A. 2005, MNRAS, 356, 1201

Bate, M. R., Bonnell, I. A., \& Bromm, V. 2002, MNRAS, 332, L65

Bate, M. R., Bonnell, I. A., \& Bromm, V. 2003, MNRAS, 339, 577

Bertin, E. 2006, ASP Conf. Ser. 351, Astronomical Data Analysis Software and Systems XV, ed. C. Gabriel, C. Arviset, D. Ponz, \& E. Solano (San Francisco, CA: ASP), 112

Bertin, E., \& Arnouts, S. 1996, A\&AS, 117, 393

Bertin, E., Mellier, Y., Radovich, M., et al. 2002, ASP Conf. Ser. 281, Astronomical Data Analysis Software and Systems XI, ed. D. A. Bohlender, D. Durand, \& T. H. Handley (San Francisco, CA: ASP), 228

Beuzit, J.-L., Feldt, M., Dohlen, K., et al. 2008, Proc. SPIE, 7014, 701418

Binks, A. S., \& Jeffries, R. D. 2014, MNRAS, 438, 11

Bonfils, X., Delfosse, X., Udry, S., et al. 2013, A\&A, 549, 109

Bonnefoy, M., Chauvin, G., Rojo, P., et al. 2010, A\&A, 512, 52

Boss, A. P. 2006, ApJ, 643, 501

Bowler, B. P., Liu, M. C., Shkolnik, E. L., \& Dupuy, T. J. 2013, ApJ, 774, 55

Bowler, B. P., Liu, M. C., Shkolnik, E. L., et al. 2012, ApJ, 753, 142

Brandeker, A., Jayawardhana, R., Khavari, P., Haisch, K. E., Jr, \& Mardones, D. 2006, ApJ, 652, 1572

Burgasser, A. J., Cruz, K. L., Cushing, M. C., et al. 2010a, ApJ, 710, 1142

Burgasser, A. J., Geballe, T. R., Leggett, S. K., Kirkpatrick, J. D., \& Golimowski, D. A. 2006a, ApJ, 637, 1067

Burgasser, A. J., Kirkpatrick, J. D., Brown, M. E., et al. 2002, ApJ, 564, 421 Burgasser, A. J., Kirkpatrick, J. D., Cruz, K. L., et al. 2006b, ApJS, 166, 585 Burgasser, A. J., Simcoe, R. A., Bochanski, J. J., et al. 2010b, ApJ, 725, 1405 Burningham, B., Leggett, S. K., Homeier, D., et al. 2011, MNRAS, 414, 3590 Caffau, E., Ludwig, H. G., Steffen, M., Freytag, B., \& Bonifacio, P. 2011, SoPh, 268,255

Chabrier, G., \& Baraffe, I. 1997, A\&A, 327, 1039

Chauvin, G., Lagrange, A. M., Dumas, C., et al. 2004, A\&A, 425, L29

Chiu, K., Fan, X., Leggett, S. K., et al. 2006, AJ, 131, 2722

Chun, M. R., Toomey, D. W., Wahhaj, Z., et al. 2008, Proc. SPIE, 7015, 49

Cushing, M. C., Marley, M. S., Saumon, D., et al. 2008, ApJ, 678, 1372

Cushing, M. C., Vacca, W. D., \& Rayner, J. T. 2004, PASP, 116, 362

Cutri, R. M., Skrutskie, M. F., van Dyk, S., et al. 2003, yCat, 2246, 0

Cutri, R. M., Wright, E. L., Conrow, T., et al. 2012, Explanatory Supplement to the WISE All-Sky Data Release Products

da Silva, L., Torres, C. A. O., de la Reza, R., et al. 2009, A\&A, 508, 833

Day-Jones, A. C., Pinfield, D. J., Ruiz, M. T., et al. 2010, MNRAS, 410, 705

Delorme, P., Gagné, J., Malo, L., et al. 2012, A\&A, 548, 26

Delorme, P., Willott, C. J., Forveille, T., et al. 2008, A\&A, 484, 469

Dhital, S., West, A. A., Stassun, K. G., \& Bochanski, J. J. 2010, AJ, 139, 2566 
Donati, J. F., Catala, C., Landstreet, J. D., \& Petit, P. 2006, in ASP Conf. Ser. 358, Solar Polarization 4, ed. R. Casini \& B. W. Lites (San Francisco, CA: ASP), 362

Donati, J. F., Semel, M., Carter, B. D., Rees, D. E., \& Collier Cameron, A. 1997, MNRAS, 291, 658

Ducourant, C., Le Campion, J. F., Rapaport, M., et al. 2006, A\&A, 448, 1235

Ducourant, C., Teixeira, R., Chauvin, G., et al. 2008, A\&A, 477, L1

Dupuy, T. J., \& Liu, M. C. 2012, ApJS, 201, 19

Faherty, J. K., Burgasser, A. J., West, A. A., et al. 2010, AJ, 139, 176

Fernández, D., Figueras, F., \& Torra, J. 2008, A\&A, 480, 735

Ftaclas, C., Martín, E. L., \& Toomey, D. 2003, IAU Symp. 211, Brown Dwarfs, ed. E. Martín (San Francisco, CA: ASP), 521

Gagné, J., Lafrenière, D., Doyon, R., Malo, L., \& Artigau, É. 2014, ApJ, 783, 121

Gardner, J. P., Mather, J. C., Clampin, M., et al. 2006, SSRv, 123, 485

Geißler, K., Metchev, S., Kirkpatrick, J. D., Berriman, G. B., \& Looper, D. 2011, ApJ, 732, 56

Gizis, J. E. 2002, ApJ, 575, 484

Goldman, B., Marsat, S., Henning, T., Clemens, C., \& Greiner, J. 2010, MNRAS, 405,1140

Hinkle, K. H., Blum, R. D., Joyce, R. R., et al. 2003, Porc. SPIE, 4834, 353

Hook, I. M., Jørgensen, I., Allington Smith, J. R., et al. 2004, PASP, 116, 425

Inaba, S., Wetherill, G. W., \& Ikoma, M. 2003, Icar, 166, 46

Irwin, J., Berta, Z. K., Burke, C. J., et al. 2011, ApJ, 727, 56

Kaeufl, H.-U., Ballester, P., Biereichel, P., et al. 2004, Proc. SPIE, 5492, 1218

Kirkpatrick, J. D., Cushing, M. C., Gelino, C. R., et al. 2011, ApJS, 197, 19

Konopacky, Q. M., Barman, T. S., Macintosh, B. A., \& Marois, C. 2013, Sci, 339, 1398

Kuzuhara, M., Tamura, M., Kudo, T., et al. 2013, ApJ, 774, 11

Lagrange, A. M., Bonnefoy, M., Chauvin, G., et al. 2010, Sci, 329, 57

Lagrange, A. M., Gratadour, D., Chauvin, G., et al. 2009, A\&A, 493, L21

Leggett, S. K., Currie, M. J., Varricatt, W. P., et al. 2006, MNRAS, 373, 781

Leggett, S. K., Saumon, D., Albert, L., et al. 2008, ApJ, 682, 1256

Liu, M. C., Delorme, P., Dupuy, T. J., et al. 2011, ApJ, 740, 108

Liu, M. C., Dupuy, T. J., \& Leggett, S. K. 2010, ApJ, 722, 311

Liu, M. C., Leggett, S. K., Golimowski, D. A., et al. 2006, ApJ, 647, 1393

Lodders, K. 2002, Icar, 155, 393

Lopez Santiago, J., Montes, D., Crespo Chacon, I., \& Fernandez Figueroa, M. J. 2006, ApJ, 643, 1160

Lowrance, P. J., McCarthy, C., Becklin, E. E., et al. 1999, ApJL, 512, L69

Luhman, K. L., Burgasser, A. J., \& Bochanski, J. J. 2011, ApJL, 730, L9

Luhman, K. L., Patten, B. M., Marengo, M., et al. 2007, ApJ, 654, 570

Luhman, K. L., Stauffer, J. R., \& Mamajek, E. E. 2005, ApJL, 628, L69

Mace, G. N., Kirkpatrick, J. D., Cushing, M. C., et al. 2013, ApJ, 777, 36

Macintosh, B. A., Anthony, A., Atwood, J., et al. 2012, Proc. SPIE, 8446, $84461 \mathrm{U}$

Malo, L., Artigau, E., Doyon, R., et al. 2014, arXiv:1402.6053

Malo, L., Doyon, R., Lafrenière, D., et al. 2013, ApJ, 762, 88

Mann, A. W., Brewer, J. M., Gaidos, E., Lépine, S., \& Hilton, E. J. 2013, AJ, 145,52

Marley, M. S., Saumon, D., \& Goldblatt, C. 2010, ApJL, 723, L117

Marois, C., Macintosh, B., Barman, T. S., et al. 2008, Sci, 322, 1348

Marois, C., Zuckerman, B., Konopacky, Q. M., Macintosh, B., \& Barman, T. S. 2010, Natur, 468, 1080

McCaughrean, M. J., Close, L., Scholz, R.-D., et al. 2004, A\&A, 413, 1029

Mentuch, E., Brandeker, A., van Kerkwijk, M. H., Jayawardhana, R., \& Hauschildt, P. H. 2008, ApJ, 689, 1127

Messina, S., Desidera, S., Turatto, M., Lanzafame, A. C., \& Guinan, E. F. 2010, A\&A, 520, 15

Metchev, S. A., \& Hillenbrand, L. A. 2006, ApJ, 651, 1166
Monet, D. G., Levine, S. E., Canzian, B., et al. 2003, AJ, 125, 984

Morley, C. V., Fortney, J. J., Marley, M. S., et al. 2012, ApJ, 756, 172

Neuhäuser, R., \& Schmidt, T. O. B. 2012, arXiv:1201.3537v1

Newton, E. R., Charbonneau, D., Irwin, J., et al. 2014, AJ, 147, 20

Norton, A. J., Wheatley, P. J., West, R. G., et al. 2007, A\&A, 467, 785

Perets, H. B., \& Kouwenhoven, M. B. N. 2012, ApJ, 750, 83

Pollack, J. B., Hubickyj, O., Bodenheimer, P., et al. 1996, Icar, 124, 62

Preibisch, T., \& Feigelson, E. D. 2005, ApJS, 160, 390

Puget, P., Stadler, E., Doyon, R., et al. 2004, Proc. SPIE, 5494, 978

Rafikov, R. R. 2009, ApJ, 704, 281

Rajpurohit, A. S., Reylé, C., Allard, F., et al. 2013, A\&A, 556, 15

Rameau, J., Chauvin, G., Lagrange, A. M., et al. 2013, ApJL, 772, L15

Rayner, J. T., Toomey, D. W., Onaka, P. M., et al. 2003, PASP, 115, 362

Reid, I. N., Hawley, S. L., \& Gizis, J. E. 1995, AJ, 110, 1838

Reipurth, B., \& Clarke, C. 2001, AJ, 122, 432

Reylé, C., Delorme, P., Willott, C. J., et al. 2010, A\&A, 522, A112

Riaz, B., Gizis, J. E., \& Harvin, J. 2006, AJ, 132, 866

Riedel, A. R., Murphy, S. J., Henry, T. J., et al. 2011, AJ, 142, 104

Roeser, S., Demleitner, M., \& Schilbach, E. 2010, AJ, 139, 2440

Roeser, S., Schilbach, E., Schwan, H., et al. 2008, A\&A, 488, 401

Rojas-Ayala, B., Covey, K. R., Muirhead, P. S., \& Lloyd, J. P. 2012, ApJ, 748, 93

Rousselot, P., Lidman, C., Cuby, J. G., Moreels, G., \& Monnet, G. 2000, A\&A, 354,1134

Saumon, D., \& Marley, M. S. 2008, ApJ, 689, 1327

Saumon, D., Marley, M. S., Abel, M., Frommhold, L., \& Freedman, R. S. 2012, ApJ, 750, 74

Saumon, D., Marley, M. S., Cushing, M. C., et al. 2006, ApJ, 647, 552

Schlieder, J. E., Lépine, S., \& Simon, M. 2012, AJ, 143, 80

Schmitt, J. H. M. M., Fleming, T. A., \& Giampapa, M. S. 1995, ApJ, 450, 392

Schneider, J., Dedieu, C., Le Sidaner, P., Savalle, R., \& Zolotukhin, I. 2011, A\&A, 532, 79

Sills, A., Pinsonneault, M. H., \& Terndrup, D. M. 2000, ApJ, 534, 335

Simons, D. A., \& Tokunaga, A. 2002, PASP, 114, 169

Spiegel, D. S., Burrows, A. S., \& Milsom, J. A. 2011, ApJ, 727, 57

Stamatellos, D., Hubber, D. A., \& Whitworth, A. P. 2007, MNRAS, 382, L30

Stamatellos, D., \& Whitworth, A. P. 2009, MNRAS, 392, 413

Stephens, D. C., Leggett, S. K., Cushing, M. C., et al. 2009, ApJ, 702, 154

Suzuki, R., Tamura, M., Suto, H., et al. 2009, in AIP Conf. Proc. 1158, Exoplanets and Disks: Their Formation and Diversity, ed. T. Usuda, M. Tamura, \& M. Ishii (Melville, NY: AIP), 293

Terrien, R. C., Mahadevan, S., Bender, C. F., et al. 2012, ApJL, 747, L38

Tokunaga, A. T., \& Vacca, W. D. 2005, PASP, 117, 421

Vacca, W. D., Cushing, M. C., \& Rayner, J. T. 2003, PASP, 115, 389

van Dam, M. A., Sasiela, R. J., Bouchez, A. H., et al. 2006, Proc. SPIE, 6272, 627231

Voges, W., Aschenbach, B., Boller, T., et al. 1999, A\&A, 349, 389

Wahhaj, Z., Liu, M. C., Biller, B. A., et al. 2011, ApJ, 729, 139

Weinberg, M. D., Shapiro, S. L., \& Wasserman, I. 1987, ApJ, 312, 367

West, A. A., Hawley, S. L., Bochanski, J. J., et al. 2008, AJ, 135, 785

White, R. J., \& Basri, G. S. 2003, ApJ, 582, 1109

Wizinowich, P. L., Chin, J., Johansson, E., et al. 2006, Proc. SPIE, 6272, 627209

Wright, E. L., Eisenhardt, P. R. M., Mainzer, A. K., et al. 2010, AJ, 140, 1868

Yee, J. C., \& Jensen, E. L. N. 2010, ApJ, 711, 303

Yurchenko, S. N., \& Tennyson, J. 2014, MNRAS, 1401, 4852

Zickgraf, F. J., Engels, D., Hagen, H. J., Reimers, D., \& Voges, W. 2003, A\&A, 406, 535

Zuckerman, B., Rhee, J. H., Song, I., \& Bessell, M. S. 2011, ApJ, 732, 61

Zuckerman, B., \& Song, I. 2004, ARA\&A, 42, 685

Zuckerman, B., Song, I., \& Bessell, M. S. 2004, ApJL, 613, L65 